\title{
LOZENGE TILINGS, GLAUBER DYNAMICS AND MACROSCOPIC SHAPE
}

\author{
BENOÎT LASLIER AND FABIO LUCIO TONINELLI
}

\begin{abstract}
We study the Glauber dynamics on the set of tilings of a finite domain of the plane with lozenges of side $1 / L$. Under the invariant measure of the process (the uniform measure over all tilings), it is well known [4] that the random height function associated to the tiling converges in probability, in the scaling limit $L \rightarrow \infty$, to a non-trivial macroscopic shape minimizing a certain surface tension functional. According to the boundary conditions the macroscopic shape can be either analytic or contain "frozen regions" (Arctic Circle phenomenon 3, 11]).

It is widely conjectured, on the basis of theoretical considerations 23, 10, partial mathematical results [25, 1] and numerical simulations for similar models ([5], cf. also the bibliography in [25, 10]), that the Glauber dynamics approaches the equilibrium macroscopic shape in a time of order $L^{2+o(1)}$. In this work we prove this conjecture, under the assumption that the macroscopic equilibrium shape contains no "frozen region".
\end{abstract}

\section{INTRODUCTION}

Random lozenge tilings and their Glauber dynamics are a very natural object in mathematical physics, probability, combinatorics and theoretical computer science. Let $\mathcal{T}_{L}$ be the triangular lattice of mesh $1 / L$ and call the union of two adjacent triangular faces a "lozenge". A region of $\mathcal{T}_{L}$ is called tileable if it can be covered by non-overlapping lozenges, so that no hole is left, cf. Figure2. Typically, the number of possible tilings of a tileable region grows like the exponential of $L^{2}$ time its area, when the lattice mesh tends to zero. To a lozenge tiling is naturally associated a height function, so that a tiling can be seen as a discrete interface, see again Figure2, When the mesh tends to zero and the height function at the boundary of the domain tends to some well-defined boundary height $\varphi$, the height function of a random tiling sampled from the uniform measure tends in probability to a certain limit shape $\bar{\phi}$. This limit shape minimizes the surface energy functional defined in formula (2), compatibly with the boundary height. According to the choice of the boundary height, $\bar{\phi}$ is either analytic, with $\nabla \bar{\phi}$ contained in the interior of a bounded set $\mathbb{T}$ of "allowed slopes" ( $\mathbb{T}$ is a triangle, see later) or it can show coexistence of analytic portions ("liquid phase") and "frozen regions" or facets where $\nabla \bar{\phi}$ is on the boundary of $\mathbb{T}$ (facets correspond microscopically to regions where at least one of the three types of lozenges has vanishing probability of being present). For special boundary heights, $\bar{\phi}$ can happen to be non-frozen and flat (with constant slope in the interior of $\mathbb{T})$. 
The Glauber dynamics on lozenge tilings is a natural Markov process whose updates consist in rotating by an angle $180^{\circ}$ three lozenges that share a vertex, see Figure 1. Such dynamics received a lot of attention in the-

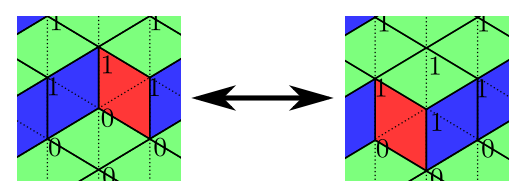

Figure 1. The allowed updates.

oretical computer science [19, 25, 24, since it is a conceptually and algorithmically simple way of sampling a random tiling in the long-time limit (the invariant measure of the process is the uniform one) ${ }^{11}$. In this context, a natural question that was investigated in the mentioned works was, how long one should run the dynamics before the uniform measure is reached. The Glauber dynamics is an even more natural stochastic process in mathematical physics: In the height function representation, lozenge dynamics is equivalent to the zero-temperature dynamics of interfaces, separating "+" and "-" spins, for the three-dimensional Ising model. Then, the question of convergence to equilibrium takes a rather different flavor: how long does it take before the interface, started far from equilibrium, approximates the macroscopic shape? Does the stochastic evolution converge to a deterministic, macroscopic evolution under suitable time rescaling?

It is widely believed that the time to reach equilibrium should scale like $L^{2}$ : actually, one expects that under diffusive scaling of time (i.e. setting $\tau=t / L^{2}$ ) the limiting deterministic evolution of the height function $\phi$ should roughly be the gradient flow associated to the surface energy functional,

$$
\frac{d}{d \tau} \phi=\mu(\nabla \phi) \mathcal{L} \phi
$$

Here $\mathcal{L}$, directly related to the first variation of the surface energy functional, is the non-linear elliptic operator defined in (4), while $\mu(\nabla \phi)$ is a "mobility coefficient". See [23] for an illuminating discussion of these issues. For $\tau \gg 1$ (i.e. $t \gg L^{2}$ ) the interface should asymptotically reach the macroscopic shape, characterized by $\mathcal{L} \bar{\phi}=0$. This belief is supported by numerical simulations (for this and related models, see [5] and references in [25, 10]), heuristic arguments [10] and partial mathematical results [25, 1].

Let us also mention that, for the zero-temperature two-dimensional (and not three-dimensional) Ising model, convergence of the evolution of spin droplets to a deterministic equation of anisotropic mean-curvature type under diffusive scaling has been achieved very recently [15, 16]. The limit equation is somewhat the analog of (1), with the notable difference that in that case $\phi$ describes a curve in the plane and not a surface in threedimensional space. What helps in the two-dimensional case is that, as observed in [23], the stochastic interface evolution can be locally mapped to well-studied interacting particle processes like one-dimensional symmetric

\footnotetext{
${ }^{1}$ Let us mention that there are alternative, algorithmically more efficient, ways to sample uniform random tilings, see for instance [26, 14] or [20].
} 
simple exclusion and zero-range processes. None of these mappings holds in the three-dimensional case (i.e. for lozenge dynamics) and a host of new ideas is called for.

The main result of the present work is a mathematical confirmation of the $L^{2}$ scaling of the equilibration time:

Theorem 1 (Informal version). If the macroscopic shape $\bar{\phi}$ contains no frozen region then, whatever the initial condition of the dynamics, at time $L^{2+o(1)}$ the height function is with high probability at distance o(1) from $\bar{\phi}$.

See Theorem 3.1 for a precise formulation. Some previous results in this direction are mentioned in Section 3.1.

It is at present unclear to us whether the restriction to non-frozen macroscopic shapes is just a technical limitation or if something deeper happens. Frozen regions reflect the singularities of the surface tension functional and it is a priori possible that such singularities might have a drastic effect on dynamics. For the two-dimensional Ising model at zero temperature, singularities of the surface tension do not modify the time scaling $L^{2}$, but they have the effect that the deterministic macroscopic interface evolution one obtains in the diffusive limit is not smooth: the curvature of the interface is in general not differentiable in space [15, Section 2.2.2].

An obvious difficulty in attacking the lozenge dynamics problem is that we have no a priori knowledge of the non-equilibrium interface fluctuations during the evolution (before the equilibrium state is reached), or even of their order of magnitude. A natural idea is to look at the system on mesoscopic regions, sufficiently small so that macroscopic properties of the interface (slope, curvature, ...) are almost constant but much larger than the lattice spacing so that statistical fluctuations are small. More precisely one might expect that, if at some time $t$ the interface approximates some smooth height function $\phi_{t}$, then locally in the neighborhood of a point where the normal vector to $\phi_{t}$ is $\mathbf{n}$, the statistics of the interface will be determined by the infinite-volume, translation invariant Gibbs state of slope $\mathbf{n}$ [13]. This reasoning would suggest height fluctuations of order $\sqrt{\log L} / L$. "Local equilibrium" ideas of this type are rather classical in physics, for the macroscopic derivation of the equations of fluid dynamics from microscopic particle systems.

In our case, this intuition seems extremely difficult to substantiate mathematically, yet we do use it somehow. Indeed the route we follow to prove Theorem 1 is to show that, if time is rescaled a bit more than diffusively (by setting $\tau=t / L^{2+\epsilon}$ with $\epsilon>0$ arbitrarily small), then the interface is bounded above and below by two evolving surfaces that follow an auxiliary deterministic equation that morally looks like (1) and both converge to the macroscopic shape. Via this auxiliary, slowed down, evolution we are able to make use of the "local equilibrium" intuition mentioned above.

We will not try to explain in detail the idea of the proof of Theorem 1 in this introduction. At this stage, let us just point out that one of the main difficulties we have to overcome is to precisely estimate average height and height fluctuations in mesoscopic regions of size $L^{-1 / 2+\delta}$ with $\delta$ small, for a rather large class of boundary heights. In [1] the analog of Theorem 1 in the 
special case where the macroscopic shape is flat (i.e. $\bar{\phi}$ is an affine function) was proven: there, the problem of controlling equilibrium fluctuations in finite domains was bypassed since it was possible to reduce to fluctuation estimates in the translation invariant infinite-volume Gibbs states mentioned above. In the present case, this trick cannot work.

In general, it is only for special domains and boundary conditions that precise estimates on height fluctuations and on the finite- $L$ corrections to the average height w.r.t. to the macroscopic limit $\bar{\phi}$ are known in the literature. See for instance [12] for domino tilings. More relevant for us are the works [21, 22] by L. Petrov. There, the author considers uniform random tilings of a hexagon $\square_{a b c}$ of sides $a, b, c$. In this case, the macroscopic shape (that is not flat) can be written down "explicitly" [3] (cf. also Sections 3.2 and 4 below) and the equilibrium measure has an exact determinantal representation [21, 22 which allows for precise asymptotic analysis.

One of the main new ideas of our work is that, locally in regions of size $\approx L^{-1 / 2+\delta}$, we can compare the height of the randomly evolving interface with the random equilibrium height of lozenge tilings in a hexagonal region $\bigcirc_{a b c}$ with suitably chosen, time-dependent parameters $a, b, c$. Technically, one key result we prove, which might be of interest by itself, is the following:

Theorem 2 (Informal version). Given a macroscopic shape $\bar{\phi}$ in a domain $U$, if $\bar{\phi}$ is smooth in the neighborhood of a point $u \in U$, then the Taylor expansion of $\bar{\phi}$ around $u$ coincides up to second order with the Taylor expansion of the macroscopic shape associated to some hexagon $\square_{a b c}$.

We will call the second-order Taylor expansion of $\bar{\phi}$ at a given point a "local structure". We would like to emphasize that Theorem 2 is a priori not obvious: As we will see in Section 4, the set of all admissible local structures associated to arbitrary macroscopic shapes is parametrized by four variables (two for the slope and two for the Hessian matrix), while "hexagonal" local structures are parametrized by a different set of four variables with a rather different meaning (two for the side-lengths of the hexagon and two for the coordinates of a point inside the hexagon). We have then to check that a certain explicit but complicated function from $\mathbb{R}^{4}$ to $\mathbb{R}^{4}$ is surjective (actually it turns out to be a bijection).

Theorem 2 would be false if "second order" were replaced, say, by "third order" (it would require surjectivity of a function from $\mathbb{R}^{4}$ to $\mathbb{R}^{8}$ ). Remarkably, for the proof of Theorem 1 the second-order comparison provided by Theorem 2 is sufficient. The basic reason is that, in regions of size $L^{-1 / 2+\delta}$, third- or higher-order terms in the expansion of the macroscopic shape give negligible contributions of order $L^{-3 / 2+3 \delta}$, much smaller than the minimal significant length-scale of the model, which is the lattice spacing $1 / L$.

\section{RANDOM LOZENGE TILINGS AND HEIGHT FUNCTION}

2.1. Monotone surfaces and height functions. Let $L$ be an integer, that will be taken large later. Closed squares in $\mathbb{R}^{3}$ of side $1 / L$, with the four vertices in $(\mathbb{Z} / L)^{3}$, will be called faces of $(\mathbb{Z} / L)^{3}$.

Definition 2.1. A discrete (or stepped) monotone surface $\Sigma_{L}$ is a connected union of faces of $(\mathbb{Z} / L)^{3}$ that projects bijectively on the 111 plane $P_{111}$. 


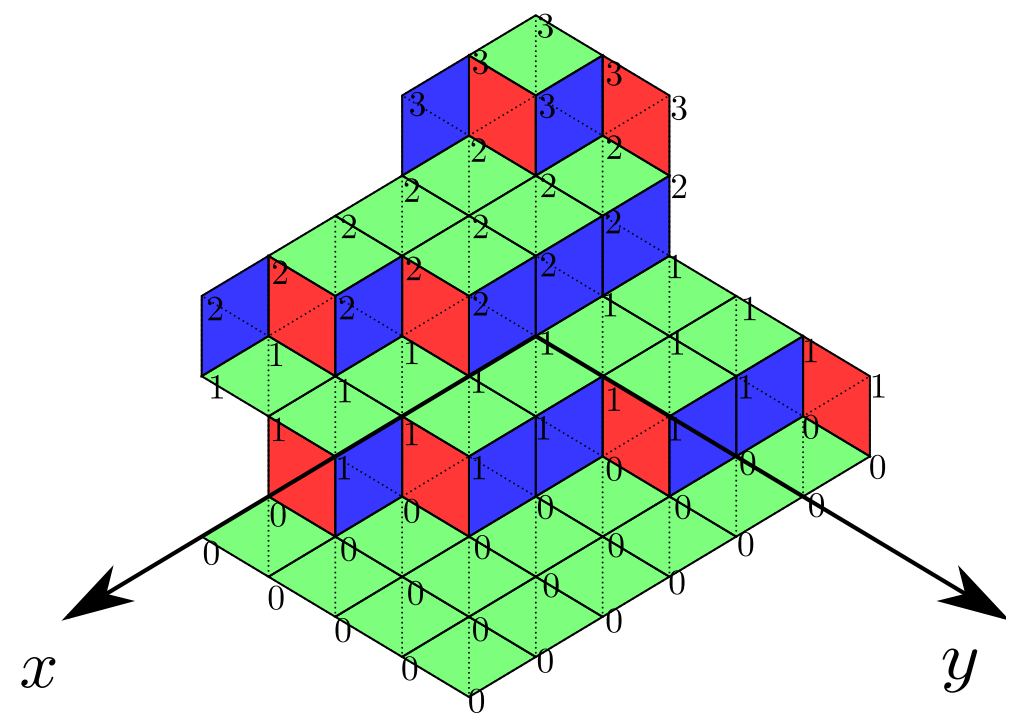

Figure 2. A portion of lozenge tiling associated to a stepped monotone interface. Next to each vertex of $\mathcal{T}_{L}$ is marked the value of the height function. The underlying triangular lattice is given by dotted lines.

Look at Figure 2 the projection of each square face of $\Sigma_{L}$ is a lozenge with angles $60^{\circ}$ and $120^{\circ}$ and three possible orientations: horizontal, south-east and south-west, according to whether the normal vector to the square face of $\Sigma_{L}$ is $(0,0,1),(1,0,0)$ or $(0,1,0)$. The projection of $\Sigma_{L}$ gives therefore a lozenge tiling of $P_{111}$ with these three types of tiles. Vertices of the lozenges are the vertices of a triangular lattice $\mathcal{T}_{L}$ of side const./L. Via a suitable choice of coordinates, we will set the constant to be 1 below.

Definition 2.2. A continuous monotone surface $\Sigma$ in $\mathbb{R}^{3}$ is a two-dimensional connected surface such that:

(1) $\Sigma$ projects bijectively on $P_{111}$;

(2) the normal vector to $\Sigma$, assumed to be defined almost everywhere, points in $\mathbb{R}_{\geq 0}^{3}$.

Note that injectivity of the orthogonal projection of $\Sigma$ on $P_{111}$ is a consequence of the assumption on the normal vector.

Definition 2.3 (Height function). To a continuous (resp. stepped) monotone surface $\Sigma$ (resp. $\Sigma_{L}$ ) we associate a height function $\phi: P_{111} \rightarrow \mathbb{R}$ (resp. $\left.h: \mathcal{T}_{L} \rightarrow \mathbb{Z} / L\right)$, as follows: $\phi(u)$ (resp. $h(u)$ ) equals the height with respect to the horizontal plane of the point $p \in \Sigma$ (resp. $p \in \Sigma_{L}$ ) whose orthogonal projection on $P_{111}$ is $u$.

Note that, for discrete monotone surfaces, heights are associated to vertices of lozenges, i.e. to vertices of $\mathcal{T}_{L}$. The definition can be extended to obtain a real-valued height function $h$ on the whole $P_{111}$, simply by establishing that the height is linear on triangular faces of $\mathcal{T}_{L}$.

On $P_{111}$ introduce a coordinate frame $(x, y)$ (see Figure 2 ) such that a given reference vertex $v_{0} \in \mathcal{T}_{L}$ has coordinates $(0,0)$ and the vertices of $\mathcal{T}_{L}$ 
that are nearest neighbors of $v_{0}$ in directions $e^{-5 i \pi / 6}$ (resp. $e^{-i \pi / 6}$ ) have coordinates $(1 / L, 0)$ (resp. $(0,1 / L))$. This choice of coordinates is convenient for stepped monotone surfaces, since the axes are along two of the directions of the triangular lattice $\mathcal{T}_{L}$. Note also that the $x$ and $y$ axes are along the $P_{111}$ projections of the directions of the usual $\hat{e}_{1}$ and $\hat{e}_{2}$ coordinate axes of $\mathbb{R}^{3}$. Whenever convenient, we will implicitly identify the plane $P_{111}$ with $\mathbb{R}^{2}$.

For continuous monotone interfaces, the condition that the normal vector points in $\mathbb{R}_{\geq 0}^{3}$ can be reformulated as follows: wherever defined, the gradient $\nabla \phi=\left(\partial_{x} \phi, \partial_{y} \phi\right)$ belongs to $\mathbb{T}$, where:

Definition 2.4. $\mathbb{T} \subset \mathbb{R}^{2}$ is the triangle with vertices $(0,0),(0,-1),(-1,0)$.

Remark 2.5. When one moves by one lattice step in $\mathcal{T}_{L}$ along the $x$ or $y$ directions the height function of a stepped interface decreases by $1 / L$ if one crosses a lozenge, and is unchanged if one moves along the edge of a lozenge. When instead one moves by a lattice step upward in the vertical direction (i.e. by $(-1 / L,-1 / L)$ in the $(x, y)$ coordinates), the height function is unchanged if one crosses a lozenge, and increases by $1 / L$ if one moves along the edge of a lozenge.

While a discrete height function uniquely identifies a lozenge tiling, in view of Remark 2.5 a lozenge tiling identifies the height function $h$ only modulo a global additive constant (the tiling identifies the height gradients). If however one fixes the height at some vertex, then the correspondence is bijective. In the following, the height along the boundary of a finite region will be fixed, so we will freely identify height functions and lozenge tilings.

Definition 2.6 (Domains). In the continuous surface setting, a domain $U$ will denote a simply connected, bounded, closed subset of $P_{111}$, whose boundary $\partial U$ is a piecewise $C^{\infty}$ simple curve.

In the discrete setting, a discrete domain $U_{L}$ will be a simply connected, bounded union of closed triangular faces of $\mathcal{T}_{L}$. With some abuse of notation, we will often identify $U_{L}$ with $U_{L} \cap \mathcal{T}_{L}$. The set of sites $v \in U_{L} \cap \mathcal{T}_{L}$ that are not on $\partial U_{L}$ is denoted $U_{L}^{\text {int }}$, while $U_{L}^{\text {ext }}=\mathcal{T}_{L} \backslash U_{L}^{\text {int }}$.

Definition 2.7 (Non-extremal monotone surface). Let $\Sigma$ be a continuous monotone surface, with height function $\phi$, and $U$ be a domain of $P_{111}$. We say that $\Sigma$ is non-extremal in $U$ if $\nabla \phi$ is defined everywhere in $\stackrel{\circ}{U}$ (the interior of $U$ ) and there exists $\epsilon>0$ such that, for every $(x, y) \in \stackrel{\circ}{U}, \nabla \phi$ is at distance at least $\epsilon$ from the boundary of the triangle $\mathbb{T}$.

In geometric terms, this means that all three components of the normal vector to $\Sigma$ are larger than a constant times $\epsilon$, at every point that projects on $\stackrel{\circ}{U}$.

Definition 2.8 (Continuous boundary heights). Given a domain $U \subset P_{111}$, a function $\varphi: P_{111} \backslash U \mapsto \mathbb{R}$ is called (continuous) boundary height if there exists a continuous monotone surface $\Sigma$ whose height function $\phi$ coincides with $\varphi$ on $P_{111} \backslash U$.

Discrete boundary heights are defined similarly: 
Definition 2.9 (Discrete boundary heights). Given a discrete domain $U_{L}$ as in Definition 2.6, we call $\varphi_{L}: U_{L}^{\text {ext }} \mapsto \mathbb{Z} / L$ a discrete boundary height if there exists a stepped monotone surface $\Sigma_{L}$ whose height coincides with $\varphi_{L}$ on $U_{L}^{e x t}$.

Remark 2.10. Boundary heights have been defined for technical reasons as height functions outside certain (continuous or discrete) domains. However, with some abuse of notation, we will often see $\varphi_{L}$ and $\varphi$ as functions on $\partial U_{L}$ and $\partial U$, respectively (instead of functions on $U^{\text {ext }}$ and $P_{111} \backslash U$ ). This makes sense because we will see (DLR equations below) that the statistical properties of the height function in a domain $U_{L}$ are determined uniquely by the height on $\partial U_{L}$.

We will be mostly interested in stepped monotone surfaces that approximate as $L \rightarrow \infty$ a continuous monotone surface:

Definition 2.11 (Discretizations). Consider a continuous monotone surface $\Sigma$, a domain $U$ and, for $L \geq 1$, stepped monotone surfaces $\Sigma_{L}$ and discrete domains $U_{L}$. We say that $\left(\Sigma_{L}, U_{L}\right)_{L \geq 1}$ is a discretization of $(\Sigma, U)$ if, for some constant $C$ independent of $L$ :

(1) the boundary $\partial U_{L}$ is within Hausdorff distance $C / L$ from $\partial U$;

(2) for every $u \in U_{L} \cap U$, one has $|h(u)-\phi(u)| \leq C / L$.

The restriction $\varphi_{L}$ of $h$ (the height function of $\Sigma_{L}$ ) to $U_{L}^{\text {ext }}$ is said to be a discretization of the boundary height $\varphi=\left.\phi\right|_{P_{111} \backslash U}$.

Given $(\Sigma, U)$, one can always find a discretization $\left(\Sigma_{L}, U_{L}\right)_{L \geq 1}$ : just take $\Sigma_{L}$ as the boundary of the union of all closed cubes with vertices in $(\mathbb{Z} / L)^{3}$ that are below $\Sigma$, and $U_{L}$ as the union of triangular faces of $\mathcal{T}_{L}$ contained in $U$.

2.2. Uniform measure, DLR equations and macroscopic shape. Given a discrete domain $U_{L}$ and a discrete boundary height $\varphi_{L}$ as in Definition 2.9. we let $\pi_{U_{L}}^{\varphi_{L}}$ denote the uniform measure over the set $\Omega_{U_{L}, \varphi_{L}}$ of all stepped monotone surfaces whose height on $U_{L}^{e x t}$ is $\varphi_{L}$ (by definition, there is at least one of them).

The measure $\pi_{U_{L}}^{\varphi_{L}}$ satisfies the so-called DLR equations. If $V_{L}$ is a subdomain of $U_{L}$, then under the law $\pi_{U_{L}}^{\varphi_{L}}$, conditioned to the event that the height on $V_{L}^{e x t}$ is a certain boundary height $\psi_{L}$, the height function in $V_{L}$ has the uniform law $\pi_{V_{L}}^{\psi_{L}}$.

The following well-known theorem states that, if the boundary condition $\varphi_{L}$ is the discretization of a continuous boundary height $\varphi$, with high probability under the uniform measure $\pi_{U_{L}}^{\varphi_{L}}$ the stepped interface $\Sigma_{L}$ approximates a certain macroscopic shape $\bar{\phi}$, that solves a variational principle.

Theorem 2.12. 4] Let $U$ and $\varphi$ be a domain and a continuous boundary height, satisfying the properties specified in Definitions 2.6 and 2.8.

(1) There exists a unique minimizer $\bar{\phi}$, among continuous monotone surfaces with boundary height $\varphi$, of the surface tension functional

$$
\Psi(\phi)=\int_{U} \sigma(s(u), t(u)) d^{2} u=\int_{U} \sigma(\nabla \phi) d^{2} u
$$


where

$$
\sigma(s, t)=-\frac{1}{\pi}[\Lambda(\pi s)+\Lambda(\pi t)+\Lambda(\pi(-1-s-t))]
$$

and

$$
\Lambda(\theta)=-\int_{0}^{-\theta} \log (2 \sin (t)) d t
$$

(observe that $-\theta \geq 0$, since $s, t,-1-s-t \leq 0$ if $(s, t) \in \mathbb{T})$.

(2) Let $\left(\varphi_{L}, U_{L}\right)_{L \geq 1}$ be a discretization of the boundary condition $(\varphi, U)$ and $\Sigma_{L}$ be distributed according to the uniform measure $\pi_{U_{L}}^{\varphi_{L}}$. Then, as $L \rightarrow \infty, \Sigma_{L}$ tends in $\pi_{U_{L}}^{\varphi_{L}}$-probability to $\bar{\phi}$ : for every $\epsilon>0$,

$$
\pi_{U_{L}}^{\varphi_{L}}\left(\exists u \in U_{L}:|h(u)-\bar{\phi}(u)| \geq \epsilon\right) \rightarrow 0 .
$$

The Euler-Lagrange equation associated to the variational principle is the non-linear elliptic PDE in "divergence form"

$$
\begin{aligned}
\mathcal{L} \bar{\phi}:=\partial_{x}\left(\partial_{s} \sigma(\nabla \bar{\phi})\right) & +\partial_{y}\left(\partial_{t} \sigma(\nabla \bar{\phi})\right) \\
& =a_{11}(\nabla \bar{\phi}) \partial_{x}^{2} \bar{\phi}+a_{22}(\nabla \bar{\phi}) \partial_{y}^{2} \bar{\phi}+2 a_{12}(\nabla \bar{\phi}) \partial_{x, y}^{2} \bar{\phi}=0
\end{aligned}
$$

with

$$
\begin{array}{r}
a_{11}(\nabla \bar{\phi})=\frac{1}{\tan \left(-\pi \partial_{x} \bar{\phi}\right)}+\frac{1}{\tan \left(\pi\left(1+\partial_{x} \bar{\phi}+\partial_{y} \bar{\phi}\right)\right)} \\
a_{22}(\nabla \bar{\phi})=\frac{1}{\tan \left(-\pi \partial_{y} \bar{\phi}\right)}+\frac{1}{\tan \left(\pi\left(1+\partial_{x} \bar{\phi}+\partial_{y} \bar{\phi}\right)\right)} \\
a_{12}(\nabla \bar{\phi})=a_{21}(\nabla \bar{\phi})=\frac{1}{\tan \left(\pi\left(1+\partial_{x} \bar{\phi}+\partial_{y} \bar{\phi}\right)\right)} .
\end{array}
$$

The matrix $\mathbf{a}(s, t)=\left\{a_{i j}((s, t))\right\}_{i, j=1,2}$ is strictly positive definite in $\stackrel{\circ}{\mathbb{T}}$, as a consequence of strict convexity of the surface tension functional $\Psi$ (positive definiteness can also be checked by hand; in particular, the determinant of $\mathbf{a}(s, t)$ is 1$)$. In $\stackrel{\circ}{\mathbb{T}}$ the matrix elements $a_{i j}((s, t))$ are analytic and the diagonal elements $a_{i i}(s, t)$ are strictly positive. When instead $(s, t)$ approaches $\partial \mathbb{T}$, the matrix $\mathbf{a}(s, t)$ becomes singular.

Assume that $\bar{\phi}$ is non-extremal in $U$ (its gradient is bounded away from the boundary of the set of allowed slopes). Then, $\bar{\phi}$ is real analytic in $\stackrel{\circ}{U}$ (see for instance [7, Ch. II.2 and Ch. VI.3]) and solves (4) everywhere in $\stackrel{\circ}{U}$.

It can however happen, even for some natural boundary conditions $(U, \varphi)$ (see Section 3.2), that in some subset $\hat{U} \subset U$ with non-empty interior the gradient $\nabla \bar{\phi}$ belongs to $\partial \mathbb{T}$. Such regions $\hat{U}$ are called frozen regions.

\section{Dynamics, CONJeCtures AND MAIn RESUlt}

The Glauber dynamics is defined as a Markov process $\left(h_{t}^{\eta}\right)_{t \geq 0}$ on the set $\Omega_{U_{L}, \varphi_{L}}$, with $\eta$ denoting the initial condition. To each site $v \in U_{L}^{i n t}$ such that all six neighbors of $v$ are in $U_{L}$, we associate a mean-one Poisson clock. Clocks at different sites are independent. When the clock at $v$ rings, if in the present lozenge configuration $v$ belongs to exactly three lozenges, then we turn the three lozenges by an angle $\pi$. See Figure 1. In terms of height function, an update corresponds to increasing by $+1 / L$ or decreasing by $-1 / L$ 
the height $h_{t}^{\eta}(v)$ with rate 1 , with the constraint that $h_{t}^{\eta}$ remains a stepped monotone surface in $\Omega_{U_{L}, \varphi_{L}}$ at all times.

We denote $\mu_{t}^{\eta}$ the law of $h_{t}^{\eta}$ and $\mathbb{P}$ the law of the entire process. The dynamics is reversible and its unique invariant measure is the uniform measure $\pi_{U_{L}}^{\varphi_{L}}$.

As we mentioned in the introduction, the dynamics is expected to converge to equilibrium in a time of order $L^{2}$ times sub-leading corrections. More precisely:

Conjecture 3.1. Let $U, \varphi$ and its discretizations $\left(U_{L}, \varphi_{L}\right)_{L \geq 1}$ be as above. For every $\delta>0$ there exists $c(\delta)<\infty$ such that, whatever the initial condition $\eta$, at times $t>c(\delta) L^{2+\delta}$ the following holds with probability tending to 1 as $L \rightarrow \infty$ : for every vertex $v \in U_{L}$

$$
\left|h_{t}^{\eta}(v)-\bar{\phi}(v)\right|=o(1) .
$$

In other words, within time $L^{2+o(1)}$ the interface macroscopically approximates the equilibrium shape to any pre-assigned precision.

Actually, we believe that more should be true: at time $L^{2+o(1)}$, the law $\mu_{t}^{\eta}$ should be very close to the equilibrium measure $\pi_{U_{L}}^{\varphi_{L}}$. More precisely, define the mixing time of the dynamics as

$$
T_{\text {mix }}=T_{\text {mix }}\left(U_{L}, \varphi_{L}\right)=\inf \left\{t: \max _{\eta}\left\|\mu_{t}^{\eta}-\pi_{U_{L}}^{\varphi_{L}}\right\| \leq 1 /(2 e)\right\},
$$

with $\|\mu-\nu\|$ the total variation distance between two probability measures $\mu, \nu$. Then:

Conjecture 3.2. In the same setting of Conjecture 3.1, it is expected that $T_{\text {mix }}=O\left(L^{2+o(1)}\right)$.

Thanks to the classical inequality

$$
\max _{\eta}\left\|\mu_{t}^{\eta}-\pi_{U_{L}}^{\varphi_{L}}\right\| \leq e^{-\left\lfloor t / T_{\text {mix }}\right\rfloor},
$$

this would say that, at time of order $L^{2+o(1)} \log (1 / \delta), \mu_{t}^{\eta}$ is within variation distance $\delta$ from equilibrium, for any arbitrary $\delta$.

Our main results is a proof of Conjecture 3.1 under the assumption that the macroscopic shape has no frozen regions (Theorem 3.1). As mentioned at the end of Section 3.2, the methods we develop in this work allow also to prove the stronger Conjecture 3.2 for a rather special class of boundary conditions (Theorem 3.6 , details will be given in a forthcoming publication).

Theorem 3.1. Let the domain $U$ and the boundary condition $\varphi$ satisfy the assumptions of Definitions 2.6 and 2.8. Assume in addition that the associated macroscopic shape $\bar{\phi}$ is non-extremal in $U$ and let $\left(\varphi_{L}, U_{L}\right)_{L \geq 1}$ be a discretization of $(\varphi, U)$. Consider the Glauber dynamics in $U_{L}$ with boundary height $\varphi_{L}$ and initial condition $\eta$. There exists a sequence $\epsilon_{L}$ tending to zero and, for each $\delta>0$, a constant $c(\delta)<\infty$ such that, with $T_{L}=c(\delta) L^{2+\delta}$,

$$
\max _{\eta} \sup _{t>T_{L}} \mathbb{P}\left(\exists v \in U_{L}:\left|h_{t}^{\eta}(v)-\bar{\phi}(v)\right| \geq \epsilon_{L}\right) \rightarrow 0 \quad \text { as } \quad L \rightarrow \infty .
$$


It is important to emphasize that, except for the $\delta$ in the exponent of $T_{L}$, this result is optimal. Indeed, it is known that there exist initial conditions $\eta$ such that, for times smaller than $a L^{2}$ with $a>0$ small (how small, depending on the domain $U$ and on the boundary height $\varphi), \max _{v}\left|h_{t}^{\eta}(v)-\bar{\phi}(v)\right|$ is still bounded away from zero. This is proven in [17, Section 10] in the special case where the macroscopic shape $\bar{\phi}$ is flat, but the proof extends with minor modifications to the case of non-extremal $\bar{\phi}$ considered here.

A slight generalization of Theorem 3.1 is the following:

Corollary 3.2. The same statement as in Theorem 3.1 holds without the assumption that $\bar{\phi}$ is non-extremal, if the following holds: there exists a sequence $\left(\varphi^{(n)}\right)_{n \geq 1}$ of continuous boundary heights on $P_{111} \backslash U$ such that:

- $\max _{x \in \partial U}\left|\varphi(x)-\varphi^{(n)}(x)\right|$ tends to zero as $n \rightarrow \infty$;

- for every $n$, the macroscopic shape $\bar{\phi}^{(n)}$ corresponding to boundary conditions $\left(U, \varphi^{(n)}\right)$ is non-extremal.

In other words, the claim of Theorem 3.1 holds if $\bar{\phi}$ can be approximated by a sequence of non-extremal macroscopic shapes. A typical application is given in Section 3.2.1 below.

3.1. Previous results. The first mathematical estimate we are aware of on the relaxation time of the Glauber dynamics for lozenge tilings is in the work of Luby, Randall and Sinclair [19], who proved rapid mixing: the mixing time grows at most like some polynomial of the graph-distance diameter of $U_{L}$ (i.e. $T_{\mathrm{mix}} \leq L^{C}$, with our notations). The estimate they obtained on $C$ was far from the expected optimal value 2 , but we emphasize that their result required essentially no conditions on the boundary height (in particular, the possible presence of frozen regions in the macroscopic shape played no role at all). A few years later, D. Wilson [25] proved the $O\left(L^{2} \log L\right)$ scaling for $T_{\text {mix }}$, but for an ad-hoc modified, highly non-local Markov dynamics introduced in [19, whose updates can modify the position of an unbounded number of lozenges at the same time. Via known comparison arguments for Markov chains [6], Wilson's result implies again [24] a non-optimal polynomial upper bound $T_{\text {mix }}=O\left(L^{6} \log L\right)$ for the mixing time of the local Glauber dynamics (actually the log factor can be removed by going through the spectral gap of the non-local chain, see [25, Section 5]).

Both [19] and [25] are based on clever path-coupling arguments: the reason why they cannot catch the right scaling $\approx L^{2}$ for the time to approach the equilibrium shape is, in our opinion, that they do not use any input from the knowledge of the macroscopic shape and of height fluctuations properties of the equilibrium measure.

A first mathematical confirmation of the $L^{2+o(1)}$ scaling for the time of convergence to equilibrium came in [1], where it was proven that $T_{\operatorname{mix}} \approx$ $L^{2+o(1)}$, under the strongly limiting assumption that the boundary height is such that the macroscopic shape $\bar{\phi}$ is flat (i.e. an affine function). The same result was proven later, with a somewhat different method, for more general tilings (e.g. domino tilings) [17].

Going beyond the flat case, as we do in the present work, requires many novel mathematical ideas. 


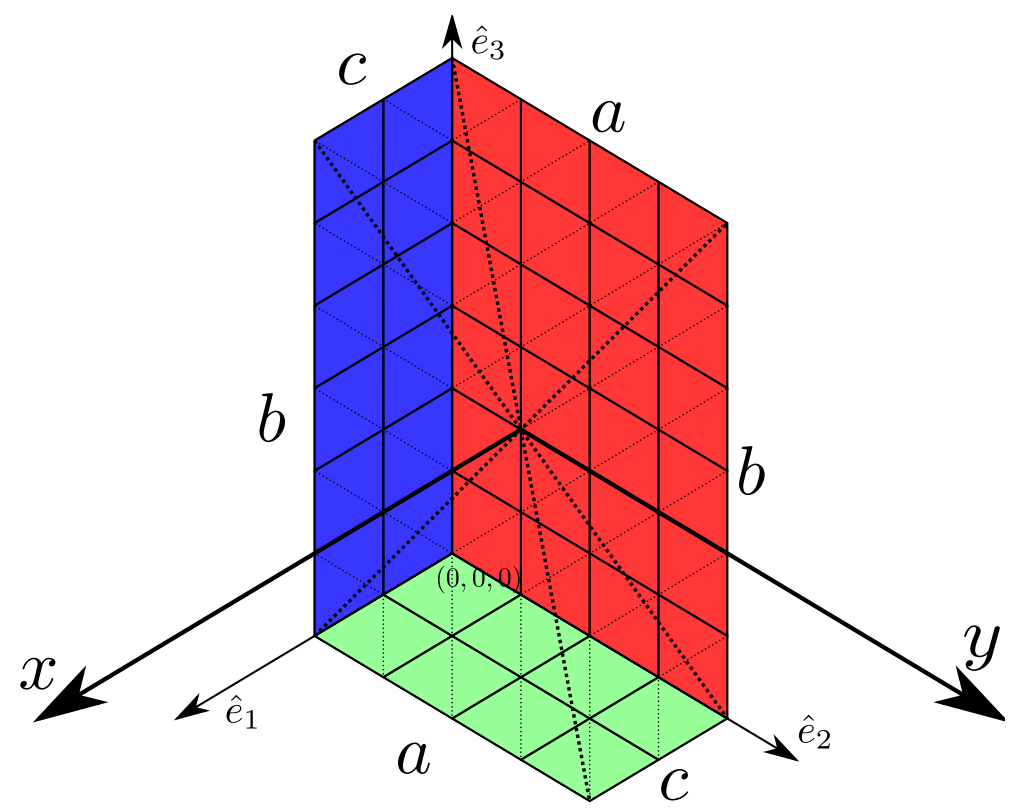

Figure 3. The hexagon $\bigcirc_{a b c}$. The origin of $(x, y)$ is set at the center of the hexagon, where the three diagonals (dotted lines) meet. The lines $\hat{e}_{i}$ are the 111 projections of the positive coordinate axes of $\mathbb{R}^{3}$.

3.2. Hexagonal regions. A crucial role in our work is played by some special boundary heights, of "hexagonal type". This may look at first surprising since the associated macroscopic shape is not at all non-extremal, in contrast with the requirements of Theorem 3.1. Such boundary conditions have played an extremely important role in the understanding of random tilings: in particular, this is the first case where the occurrence of frozen region and of the "arctic circle phenomenon" was discovered [3] (see also the earlier work [11] for domino tilings). Also, the uniform law on lozenge tilings has in this case an explicit determinantal representation. This allows to extract sharp estimates, as $L \rightarrow \infty$, on height fluctuations, on the finite-size corrections to the average height with respect to the macroscopic shape $\bar{\phi}$, and to prove convergence of height fluctuations to the Gaussian Free Field [21, 22].

Let the monotone surface $\Sigma$ be the boundary of $\left(\mathbb{R}^{+}\right)^{3}$, the positive octant of $\mathbb{R}^{3}$ and let the half-infinite lines $\hat{e}_{1}, \hat{e}_{2}, \hat{e}_{3}$ be the 111 projection of the positive coordinate axes of $\mathbb{R}^{3}$, see Figure 3 . Given $a, b, c>0$ let $\bigcirc_{a b c}$ be the hexagon in $P_{111}$ with angles of $120^{\circ}$ and with sides $a, b, c, a, b, c$, such that three of the vertices are on the lines $\hat{e}_{i}$. The sides of length $a$ are parallel to the $y$ axis and those of length $c$ to the $x$ axis (recall that the $x$ and $y$ axes are not orthogonal). See again Figure 3 . Without loss of generality, we will assume that $a+b+c=1$.

We let $\bigcirc_{a b c}$ be the open ellipse inscribed in $\bigcirc_{a b c}$, and $\varphi_{a b c}$ the boundary height of $\Sigma$ restricted to $\partial \bigcirc_{a b c}$.

This is the prototypical case where the macroscopic shape contains frozen regions: 
Theorem 3.3. [3] Call $\bar{\phi}_{a b c}$ the macroscopic shape when $U=\bigcirc_{a b c}$ and $\varphi=$ $\varphi_{a b c}$. In $\bigcirc_{a b c}, \bar{\phi}_{a b c}$ is analytic and its gradient $\nabla \bar{\phi}_{a b c}$ is in $\stackrel{\circ}{\mathbb{T}}$. On $\bigcirc_{a b c} \backslash \bigcirc_{a b c}$, $\nabla \bar{\phi}_{a b c} \in \partial \mathbb{T}$. More precisely, remove from $\bigcirc_{a b c} \backslash \bigcirc_{a b c}$ the six points of contact between $\bigcirc_{a b c}$ and the boundary of the hexagon and consider the six connected components of the set thus obtained. On the components that touch sides ac (resp. ab, resp. bc) the gradient is $(0,0)$ (resp. $(-1,0)$, resp. $(0,-1)$ ).

The ellipse $\bigcirc_{a b c}$ is called the "smooth region" or "liquid region", while $\bigcirc_{a b c} \backslash \bigcirc_{a b c}$ is the "frozen region".

A discretization of $\left(\bigcirc_{a b c}, \varphi_{a b c}\right)$ is simply obtained as $\left(\bigcirc_{a_{L} b_{L} c_{L}}, \varphi_{a_{L} b_{L} c_{L}}\right)_{L \geq 1}$ where

$$
a_{L}=(1 / L)\lfloor a L\rfloor, b_{L}=(1 / L)\lfloor b L\rfloor, c_{L}=(1 / L)\lfloor c L\rfloor .
$$

To simplify formulas we will always pretend that $a L, b L, c L$ are even integers, in which case $\bigcirc_{a_{L} b_{L} c_{L}}$ and $\varphi_{a_{L} b_{L} c_{L}}$ exactly coincide with $\oslash_{a b c}, \varphi_{a b c}$, and the center of the hexagon is a vertex of $\mathcal{T}_{L}$, that will be chosen by convention as the origin of $P_{111}$. For lightness of notations, we write simply $\pi_{L}^{a b c}$ for the uniform measure $\pi_{\bigcirc_{a_{L} b_{L} c_{L}}}^{\varphi_{a_{a} b^{c} c}}$

We already know from Theorem 2.12 that, under the measure $\pi_{L}^{a b c}$, the typical height function is macroscopically close to $\bar{\phi}_{a b c}$. The following theorem makes this claim much sharper, but the statements hold only in the liquid region:

Theorem 3.4. For every $v, u \in \bigcirc_{a b c}$

$$
\left|\left(\pi_{L}^{a b c}(h(u))-\pi_{L}^{a b c}(h(v))\right)-\left(\bar{\phi}_{a b c}(u)-\bar{\phi}_{a b c}(v)\right)\right| \leq K|u-v| / L
$$

where $K=K(a, b, c, u, v)$ is bounded as long as

$$
\min \left(a, b, c, \operatorname{dist}\left(u, \partial \bigcirc_{a b c}\right), \operatorname{dist}\left(v, \partial \bigcirc_{a b c}\right)\right)
$$

is bounded away from zero.

For every $u \in \bigcirc_{a b c}, n>0$ and $\epsilon>0$,

$$
\pi_{L}^{a b c}\left(\left|h(u)-\pi_{L}^{a b c}(h(u))\right|>L^{-1+\epsilon}\right)=O\left(L^{-n}\right)
$$

(again, the error term is uniform in $a, b, c, u$ if $\min \left(a, b, c, \operatorname{dist}\left(u, \partial \bigcirc_{a b c}\right)\right.$ ) is bounded away from zero).

The first claim is proven in Appendix A, following methods of [21, 22]. The second one follows directly from [22, Lemma 5.6], where it is proven that $\pi_{L}^{a b c}\left(L^{n}\left|h(v)-\pi_{L}^{a b c}(h(v))\right|^{n}\right)=O\left(L^{\epsilon}\right)$, plus Tchebyshev's inequality. Uniformity of the error term is not stated explicitly in [22], but it can be easily extracted from the proof).

Let us state and prove a simple consequence of Theorem 3.4 , that we need in Section 7

Proposition 3.5. Let as above $a, b, c>0$ with $a+b+c=1$ and $D_{L}$ be $a$ discrete domain contained in $\bigcirc_{a b c}$, whose distance from $\partial \bigcirc_{a b c}$ is at least $\delta>0$ independent of $L$. Let further $\varphi_{L}$ be a boundary height on $\partial D_{L}$ such that, for some $C>0$,

$$
\left|\varphi_{L}(v)-\pi_{L}^{a b c}(h(v))\right| \leq C / L \quad \text { for every } \quad v \in \partial D_{L} .
$$


Then, for every $\epsilon>0$ and $n<\infty$,

$$
\sup _{v \in D_{L}} \pi_{D_{L}}^{\varphi_{L}}\left(\left|h(v)-\pi_{L}^{a b c}(h(v))\right| \geq L^{-1+\epsilon}\right) \leq c(\delta, n, \epsilon) L^{-n} .
$$

Proof of Proposition 3.5. The arguments are rather standard, so let us be sketchy. Suppose for instance we want to upper bound

$$
\sup _{v} \pi_{D_{L}}^{\varphi_{L}}\left(h(v)-\pi_{L}^{a b c}(h(v)) \geq L^{-1+\epsilon}\right) .
$$

Define $\varphi_{L}^{\prime}=\varphi_{L}-L^{-1+\epsilon} / 2$. From 13 we see that, under the measure $\pi_{L}^{a b c}$, except with probability $O\left(L^{-n}\right)$ one has $h(v) \geq \varphi_{L}^{\prime}$ for every $v \in \partial D_{L}$. Therefore,

$$
\begin{gathered}
\sup _{v} \pi_{D_{L}}^{\varphi_{L}}\left(h(v)-\pi_{L}^{a b c}(h(v)) \geq L^{-1+\epsilon}\right)=\sup _{v} \pi_{D_{L}}^{\varphi_{L}^{\prime}}\left(h(v)-\pi_{L}^{a b c}(h(v)) \geq L^{-1+\epsilon} / 2\right) \\
\leq \sup _{v} \pi_{L}^{a b c}\left(h(v)-\pi_{L}^{a b c}(h(v)) \geq L^{-1+\epsilon} / 2\right)+O\left(L^{-n}\right) .
\end{gathered}
$$

In the last step we used monotonicity (the increasing event $h(v)-\pi_{L}^{a b c}(v) \geq$ $L^{-1+\epsilon} / 2$ becomes more likely if we replace $\varphi_{L}^{\prime}$ with a higher boundary condition, see Section 5) and the DLR equations. Then, Eq. (13) implies directly 15. (estimates are uniform in $v$ because we assumed that all $v \in D_{L}$ are uniformly bounded away, by at least $\delta$, from $\partial \bigcirc_{a b c}$.)

3.2.1. Dynamics with hexagonal boundary height. Let $U=\bigcirc_{a b c}$ and the boundary condition $\varphi$ be the restriction of $\bar{\phi}_{a b c}$ to $\partial U$. The macroscopic shape $\bar{\phi}$ is not non-extremal in $U$ since, while the gradient $\nabla \bar{\phi}$ is well-defined and belongs to $\stackrel{\circ}{\mathbb{T}}$ everywhere in $\stackrel{\circ}{U}$, it approaches $\partial \mathbb{T}$ when the boundary of $U$ is approached. However, Corollary 3.2 is applicable in this case, implying the estimate (11) on the time when the equilibrium shape is reached. Just take some sequence $u^{(n)}>0$ tending to zero, define

$$
a^{(n)}=a\left(1+u^{(n)}\right), b^{(n)}=b\left(1+u^{(n)}\right), c^{(n)}=c\left(1+u^{(n)}\right)
$$

and let $\varphi^{(n)}$ be the restriction to $\partial \bigcirc_{a b c}$ of the macroscopic shape $\bar{\phi}_{a^{(n)} b^{(n)} c^{(n)}}$ corresponding to the expanded hexagon $\bigcirc_{a^{(n)} b^{(n)} c^{(n)}}$. Since $\bigcirc_{a^{(n)} b^{(n)} c^{(n)}}$ contains $U=\bigcirc_{a b c}$ strictly, the macroscopic shape $\bar{\phi}^{(n)}=\bar{\phi}_{a^{(n)} b^{(n)} c^{(n)}}$ is nonmaximal in $U$.

The sharp control of the equilibrium measure provided by Theorem 3.4 . together with the methods developed in the proof of Theorem 3.1, allow to prove the stronger result $T_{\text {mix }} \approx L^{2+o(1)}$ (Conjecture 3.2) in the case where $U$ is a closed, simply connected subset of the open ellipse $\bigcirc_{a b c}$ and the boundary height $\varphi$ is the restriction of $\bar{\phi}_{a b c}$ to $\partial U$.

Theorem 3.6. [18] Fix $a, b, c$ with $a+b+c=1$ and $a>0, b>0, c>0$. Let $U$ be a closed domain contained in $\bigcirc_{a b c}$. Let $\varphi$ be the restriction of $\bar{\phi}_{a b c}$ to $\partial U$ and let $\left(\varphi_{L}, U_{L}\right)_{L \geq 1}$ a discretization of $(\varphi, U)$. Then, $T_{\operatorname{mix}} \leq c(\delta) L^{2+\delta}$ for every $\delta>0$. 


\section{LOCAL STRUCTURES OF MACROSCOPIC SHAPES}

In this section we formalize and prove Theorem 2 .

Let $\bar{\phi}$ be the macroscopic shape in some domain $U$ with some boundary height $\varphi$. Consider a point $(x, y) \in \stackrel{\circ}{U}$ where $\bar{\phi}$ is at least twice differentiable, and such that $\nabla \bar{\phi} \in \mathbb{O}$; let $H^{\bar{\phi}}$ be the $2 \times 2$ Hessian matrix of $\bar{\phi}$ at $(x, y)$. We call $\left\{\nabla \bar{\phi}, H^{\bar{\phi}}\right\}$, the local structure of $\bar{\phi}$ at $(x, y)$. We are excluding points $(x, y)$ where the gradient of $\bar{\phi}$ is in $\partial \mathbb{T}$, or where $\bar{\phi}$ is non-smooth: in any case, our Theorem 3.1 involves only domains where the macroscopic shape is non-extremal and in particular is $C^{\infty}$.

Recall that, if $\nabla \bar{\phi} \in \stackrel{\circ}{\mathbb{T}}$, the components of the Hessian $H^{\bar{\phi}}$ verify Eq. (4), i.e. for any local structure $\nabla \bar{\phi}$ and $H^{\bar{\phi}}$ are related by

$$
\sum_{i, j=1}^{2} a_{i j}(\nabla \bar{\phi}) H_{i j}^{\bar{\phi}}=0
$$

Therefore, to identify a local structure it is sufficient to know the gradient of $\bar{\phi}$ and two elements of the Hessian matrix, say the $\partial_{x}^{2}$ and $\partial_{x y}^{2}$ components (i.e. the (11) and $(12)=(21)$ matrix elements). In view of this, we define

$$
\mathcal{A}=\left\{z=\left(z_{1}, z_{2}, z_{11}, z_{12}\right) \in \mathbb{R}^{4}:\left(z_{1}, z_{2}\right) \in \stackrel{\circ}{\mathbb{T}}\right\}
$$

which should be seen as the set of all a priori admissible local structures. Note that it is not guaranteed that every $z \in \mathcal{A}$ can be actually realized as the local structure for some boundary condition.

Let us also define the open set

$$
W=\left\{w=(a, b, x, y) \in \mathbb{R}^{4}: a>0, b>0, a+b<1,(x, y) \in \bigcirc_{a b c}\right\}
$$

where as usual it is understood that $c=c(a, b)=1-a-b$. This is the set parametrizing points in ellipses of the type $\bigcirc_{a b c}$.

Remark 4.1. The allowed values of $(a, b)$ belong to the interior of triangle $\mathbb{V}=-\mathbb{T}$, with $\mathbb{T}$ as in Definition 2.4.

We introduce a map $f: W \mapsto \mathcal{A}$ as follows:

$$
f(a, b, x, y)=z=\left(z_{1}, z_{2}, z_{11}, z_{12}\right) \in \mathbb{R}^{4}
$$

with $\left(z_{1}, z_{2}\right)$ the slope $\nabla \bar{\phi}_{a b c}$ at $(x, y)$ (with $\bar{\phi}_{a b c}$ the macroscopic shape corresponding to the hexagon $\bigcirc_{a b c}$, as in Theorem 3.3) and

$$
\left(z_{11}, z_{12}\right)=\left(\partial_{x}^{2} \bar{\phi}_{a b c}, \partial_{x y}^{2} \bar{\phi}_{a b c}\right) \in \mathbb{R}^{2}
$$

with the derivatives computed at $(x, y)$. Note that $f(W)$ is the set of all local structures arising from macroscopic shapes with boundary heights of "hexagonal type". A priori it could be that $f(W)$ is a proper subset of $\mathcal{A}$, and even that $f(W)$ has topological dimension smaller than 4 . Indeed, hexagonal boundary conditions look very special in the class of all admissible boundary heights. However, Theorems 4.2 and 4.4 below exclude these possibilities. 
Let the $4 \times 4$ matrix

$$
D f(w)=\left(\begin{array}{cccc}
\partial_{a} z_{1} & \partial_{b} z_{1} & \partial_{x} z_{1} & \partial_{y} z_{1} \\
\partial_{a} z_{2} & \partial_{b} z_{2} & \partial_{x} z_{2} & \partial_{y} z_{2} \\
\partial_{a} z_{11} & \partial_{b} z_{11} & \partial_{x} z_{11} & \partial_{y} z_{11} \\
\partial_{a} z_{12} & \partial_{b} z_{12} & \partial_{x} z_{12} & \partial_{y} z_{12}
\end{array}\right)
$$

denote the derivative of $f$ at $w \in W$. If, for some $w \in W, D f(w)$ has (maximal) rank 4 , then $f$ is locally bijective on $\mathcal{A}$ : every point $z$ in a suitable neighborhood $B(f(w), \epsilon) \cap \mathcal{A}$ (with $B\left(z_{0}, r\right)$ the ball of radius $r$ centered at $z_{0}$ ) has a unique pre-image through $f$ in $W$ at distance $O(\epsilon)$ from $w$. We have

Theorem 4.2. The rank of $D f(w)$ is 4 for every $w \in W$. More precisely, in compact subsets of $W$ the determinant of $D f(w)$ is strictly negative.

Proof of Theorem 4.2. Call simply $\bar{\phi}$ the macroscopic shape in the $a b c$ hexagon and recall that, here and in the following, $c=1-a-b$. The explicit expression for $\nabla \bar{\phi}$ is given in []$^{2}$. Introduce the coordinates $(u, v)$ and $\left(u^{\prime}, v^{\prime}\right)$ as

$$
\begin{gathered}
u=u(x, y)=-x+\frac{y}{2}, \quad v=v(x, y)=-\frac{\sqrt{3}}{2} y \\
u^{\prime}=u^{\prime}(x, y)=y-\frac{x}{2}=u / 2-\frac{\sqrt{3}}{2} v, \quad v^{\prime}=v^{\prime}(x, y)=-\frac{\sqrt{3}}{2} x=\frac{\sqrt{3}}{2} u+\frac{v}{2} .
\end{gathered}
$$

What one finds is then

$$
\begin{gathered}
z_{1}=\partial_{x} \bar{\phi}=-\frac{1}{\pi} \cot ^{-1}\left[\frac{Q_{b c a}(u(x, y), v(x, y))}{\sqrt{E_{b c a}(u(x, y), v(x, y))}}\right] \\
z_{2}=\partial_{y} \bar{\phi}=-\frac{1}{\pi} \cot ^{-1}\left[\frac{Q_{c a b}\left(u^{\prime}(x, y), v^{\prime}(x, y)\right)}{\sqrt{E_{c a b}\left(u^{\prime}(x, y), v^{\prime}(x, y)\right)}}\right] \\
Q_{a b c}(u, v)=\frac{\sqrt{3}}{2}\left(\frac{4}{3} v^{2}-4 u^{2}+b^{2}+a b+b c-a c\right), \\
E_{a b c}(u, v)=3 a b c-\left(3(a+c)^{2} u^{2}-2 \sqrt{3}(a+2 b+c)(a-c) u v\right. \\
\left.+\left((a+2 b+c)^{2}-4 a c\right) v^{2}\right) .
\end{gathered}
$$

For later convenience, let us point out also that

$$
z_{3}:=-1-z_{1}-z_{2}=-\frac{1}{\pi} \cot ^{-1}\left[\frac{Q_{a b c}\left(u^{\prime \prime}(x, y), v^{\prime \prime}(x, y)\right)}{\sqrt{E_{a b c}\left(u^{\prime \prime}(x, y), v^{\prime \prime}(x, y)\right)}}\right]
$$

with

$$
u^{\prime \prime}(x, y)=\frac{1}{2} x+\frac{1}{2} y=-\frac{u}{2}-\frac{\sqrt{3}}{2} v, \quad v^{\prime \prime}(x, y)=-\frac{\sqrt{3}}{2} x+\frac{\sqrt{3}}{2} y=\frac{\sqrt{3}}{2} u-\frac{v}{2} \text {. }
$$

Let also $z_{11}=\partial_{x} z_{1}, z_{12}=\partial_{y} z_{1}=\partial_{x} z_{2}$.

Remark 4.3. The boundary of the ellipse $\bigcirc a b c$ corresponds to the set of zeros of $E_{b c a}(u(x, y), v(x, y))$. One can also check that

$$
E_{b c a}(u(x, y), v(x, y))=E_{c a b}\left(u^{\prime}(x, y), v^{\prime}(x, y)\right)=E_{a b c}\left(u^{\prime \prime}(x, y), u^{\prime \prime}(x, y)\right)
$$

\footnotetext{
${ }^{2}$ The authors of [3] consider on $P_{111}$ an orthogonal coordinate frame $(u, v)$ that does not coincide with the non-orthogonal coordinate frame $(x, y)$ we adopt here. The change of coordinates is given in 20, .
} 
and formulas above are understood to hold inside $\bigcirc$ abc. From the explicit formulas (19) and (22) one sees that both $f(w)$ and $D f(w)$ are $C^{\infty}$ in $W$, with uniform bounds when $w$ is in compact subsets of $W$.

We have to prove that the determinant of the matrix $D f$ is negative. Observe that, when we take derivatives with respect to $a$ or $b$, we have to remember that $c$ is a function of $a, b$. One can painfully check ${ }^{3}$ that the determinant of $D f$ equals

$$
\begin{gathered}
\operatorname{det}(D f)=\frac{1}{32 \pi^{4}} \frac{N(x, y)}{D(x, y)} \\
N(x, y)=(1-a)(1-b)(1-c)+2\left(1-a^{2}\right) y^{2}+2\left(1-c^{2}\right) x^{2}-4(1-a)(a+b) x y \\
D(x, y)=\left(y^{2}-\frac{(a+b)^{2}}{4}\right)\left(x^{2}-\frac{(1-a)^{2}}{4}\right)^{2} \\
\times\left(x-\left(y-\frac{1-b}{2}\right)\right)^{2}\left(x-\left(y+\frac{1-b}{2}\right)\right)^{2}
\end{gathered}
$$

One easily sees that $D(x, y)$ vanishes exactly along the sides of the hexagon $\bigcirc_{a b c}$, and is negative inside the hexagon (since in the hexagon the $y$ coordinate ranges between $-(a+b) / 2$ and $+(a+b) / 2)$. As for the numerator, it vanishes for

$$
y=-\frac{2(1-a)(a+b) x \pm \sqrt{2} \sqrt{(1-a)(1-b)(a+b)\left(-1+a^{2}-4 x^{2}\right)}}{2\left(a^{2}-1\right)}
$$

Since $a<1$, the square root is imaginary and therefore the numerator has no zeros. The numerator is clearly positive for $(x, y)=(0,0)$, so it is positive everywhere.

A key point for the following is that $f: W \mapsto \mathcal{A}$ is actually a bijection:

Theorem 4.4. The application $f$ is a diffeomorphism from $W$ to $\mathcal{A}$. In particular, $f(W)=\mathcal{A}$.

The non-trivial step is to prove that points on the boundary of $W$ are mapped through $f$ to points on the boundary of $\mathcal{A}$ (Proposition 4.5). Given this, the proof of Theorem 4.4 follows rather closely that of a theorem of Hadamard [9], that gives a necessary and sufficient condition for a smooth map from $\mathbb{R}^{n}$ to $\mathbb{R}^{n}$ to be a diffeomorphism, cf. for instance [8].

Proposition 4.5 (Compact sets have compact pre-images). Let $\left\{w_{n}\right\}_{n \geq 1}$ be a sequence of points in $W$, that tends as $n \rightarrow \infty$ to a point $\bar{w}$ on the boundary of $W$. Then, none of the sub-sequential limits of the sequence $\left\{f\left(w_{n}\right)\right\}_{n \geq 1}$ is in $\mathcal{A}$.

Proof of Proposition 4.5. Recall that, for $w \in W$, we write $w=(a, b, x, y)$ and $f(w)=\left(z_{1}, z_{2}, z_{11}, z_{12}\right)$. Note that

$$
\partial W=\{w:(a, b) \in \partial \mathbb{V}\} \cup\left\{w:(a, b) \in \stackrel{\circ}{\mathbb{V}},(x, y) \in \partial \bigcirc_{a b c}\right\} .
$$

\footnotetext{
${ }^{3}$ It is immediate from Eqs. 22 that the matrix elements of $D f$, and therefore also the determinant, are rational functions of $a, b, x, y$. For the actual computation of the coefficients of the two polynomials we used Mathematica, in order to symbolically simplify otherwise intractable expressions.
} 
Therefore, if $\bar{w}=(\bar{a}, \bar{b}, \bar{x}, \bar{y})=\lim _{n} w_{n} \in \partial W$, exactly one of these two conditions holds:

(A) $(\bar{a}, \bar{b}) \in \mathbb{V}$ and $(\bar{x}, \bar{y})$ is on the boundary of $\bigcirc_{\bar{a} \bar{b} \bar{c}}$;

(B) $(\bar{a}, \bar{b})$ is in $\partial \mathbb{V}$.

We have to prove that, in both cases, at least one of the following two options occurs:

(Option 1) $\left(z_{1}, z_{2}\right)$ approaches the boundary of $\mathbb{T}$ as $n \rightarrow \infty$;

(Option 2) the directional derivative $\left|\partial_{v} z_{i}\right|$ diverges as $n \rightarrow \infty$, for some $i=1,2,3$ and for some direction $v$ in the plane. This implies that either $\left(z_{1}, z_{2}\right)$ approaches $\partial \mathbb{T}$, or $z_{11}^{2}+z_{12}^{2}$ diverges, in both cases implying the statement of the Proposition.

Indeed, recall that $z_{3}=-1-z_{1}-z_{2}$ and observe that $\partial_{v} z_{i}$ is a linear combination of $\partial_{x} z_{i}$ and $\partial_{y} z_{i}$. If $\left|\partial_{v} z_{i}\right|$ diverges, then one among $z_{11}=\partial_{x} z_{1}, z_{12}=\partial_{x} z_{2}=\partial_{y} z_{1}$ or $\partial_{y} z_{2}$ diverge. If either $z_{11}$ or $z_{12}$ diverges, we are done. So suppose instead that $\partial_{y} z_{2}$ diverges. Remember that

$$
\begin{aligned}
a_{11}\left(z_{1}, z_{2}\right) \partial_{x} z_{1} & +2 a_{12}\left(z_{1}, z_{2}\right) \partial_{x} z_{2}+a_{22}\left(z_{1}, z_{2}\right) \partial_{y} z_{2} \\
& =a_{11}\left(z_{1}, z_{2}\right) z_{11}+2 a_{12}\left(z_{1}, z_{2}\right) z_{12}+a_{22}\left(z_{1}, z_{2}\right) \partial_{y} z_{2}=0
\end{aligned}
$$

and that, when $\left(z_{1}, z_{2}\right)$ is bounded away from $\partial \mathbb{T}, a_{i j}$ are finite and $a_{i i}$ are strictly positive. As a consequence, if $\partial_{y} z_{2}$ diverges then either $\left(z_{1}, z_{2}\right)$ approaches $\partial \mathbb{T}$ or at least one among $z_{11}$ and $z_{12}$ also diverges.

In Case (A), it follows directly from Theorem 3.3 that the slope $\left(z_{1}, z_{2}\right)$ approaches the boundary of $\mathbb{T}$, so Option 1 occurs.

In Case (B) we have to go back to formulas (22)- 26 ) for $z_{1}, z_{2}$ and $z_{3}=$ $-1-z_{1}-z_{2}$, that we rewrite compactly as

$$
z_{i}(x, y)=-\frac{1}{\pi} \cot ^{-1}\left[\frac{Q_{a b c}^{(i)}(x, y)}{\sqrt{E_{a b c}^{(i)}(x, y)}}\right], \quad i=1,2,3
$$

(recall that actually $E_{a b c}^{(i)}$ does not depend on $i$, so we will just write $E_{a b c}$ ). The numerators $Q_{a b c}^{(i)}$ are second-order polynomials in $x, y$, symmetric under $(x, y) \leftrightarrow(-x,-y)$. Let $Z_{a b c}^{(i)}, i=1,2,3$ be the respective level-zero sets on the $(x, y)$ plane: they are hyperbolas, that can be degenerate (two straight lines intersecting at $(0,0))$ for particular values of $a, b, c=1-a-b$. More precisely, $Z_{a b c}^{(i)}$ is degenerate if and only if $Q_{a b c}^{(i)}(0,0)=0$. It is however easy to check that there are no values $a, b, c=1-a-b$ for which the three hyperbolas are simultaneously degenerate: an explicit calculation shows that $\sum_{i=1}^{3} Q_{a b c}^{(i)}(0,0)$ is never zero. Therefore, for $n \rightarrow \infty$, at least one of the curves $Z_{a_{n} b_{n} c_{n}}^{(i)}$ tends to a non-degenerate hyperbola. To fix ideas, let us assume that this is the case for $i=1$, i.e. that

$$
\liminf _{n}\left|Q_{a_{n} b_{n} c_{n}}^{(1)}(0,0)\right|>0 .
$$

Then we proceed as follows. We first note that the sup-norm of $E_{a_{n} b_{n} c_{n}}$ on $\bigcirc_{a_{n} b_{n} c_{n}}$ is just $\delta_{n}:=E_{a_{n} b_{n} c_{n}}(0,0)=3 a_{n} b_{n} c_{n}=o(1)$ (the graph of $E_{a b c}$ is a 
concave paraboloid with gradient zero at $(0,0)$ and vanishes at the boundary of $\left.\bigcirc_{a b c}\right)$. The fact that $a_{n} b_{n} c_{n}=o(1)$ is because when $\left(a_{n}, b_{n}\right)$ approaches $\partial \mathbb{V}$, at least one of the three values $a_{n}, b_{n}, 1-a_{n}-b_{n}$ approaches zero. Given $\left(x_{n}, y_{n}\right) \in \bigcirc_{a_{n} b_{n} c_{n}}$, if for some $i=1,2,3$

$$
\left|\frac{Q_{a_{n} b_{n} c_{n}}^{(i)}\left(x_{n}, y_{n}\right)}{\sqrt{E_{a_{n} b_{n} c_{n}}\left(x_{n}, y_{n}\right)}}\right| \geq \frac{1}{\delta_{n}^{1 / 8}}
$$

then, for $n \rightarrow \infty, z_{i}$ approaches either 0 or 1 (according to the sign of $\left.Q_{a_{n} b_{n} c_{n}}^{(i)} / \sqrt{E_{a_{n} b_{n} c_{n}}}\right)$, and therefore Option 1 occurs.

Assume instead that at $\left(x_{n}, y_{n}\right)$ one has

$$
\max _{i}\left|Q_{a_{n} b_{n} c_{n}}^{(i)} / \sqrt{E_{a_{n} b_{n} c_{n}}}\right| \leq \frac{1}{\delta_{n}^{1 / 8}} .
$$

Note that automatically $\left(x_{n}, y_{n}\right)$ is bounded away from $(0,0)$, otherwise condition (36) would be violated, since one would have

$$
\left|\frac{Q_{a_{n} b_{n} c_{n}}^{(1)}\left(x_{n}, y_{n}\right)}{\sqrt{E_{a_{n} b_{n} c_{n}}\left(x_{n}, y_{n}\right)}}\right| \geq \frac{\left|Q_{a_{n} b_{n} c_{n}}^{(1)}\left(x_{n}, y_{n}\right)\right|}{\sqrt{\delta_{n}}} \simeq \frac{\left|Q_{a_{n} b_{n} c_{n}}^{(i)}(0,0)\right|}{\sqrt{\delta_{n}}}
$$

and $Q_{a_{n} b_{n} c_{n}}^{(1)}(0,0)$ is bounded away from zero for $n \rightarrow \infty$, cf. (35). We look at the derivative of $z_{i}$ at $\left(x_{n}, y_{n}\right)$ in the direction $v$ tangent to the local level line of $E_{a_{n} b_{n} c_{n}}$ : we get

$$
\partial_{v} z_{i}=-\frac{1}{\pi} \frac{1}{1+\left(Q_{a_{n} b_{n} c_{n}}^{(i)} / \sqrt{E_{a_{n} b_{n} c_{n}}}\right)^{2}} \frac{\partial_{v} Q_{a_{n} b_{n} c_{n}}^{(i)}}{\sqrt{E_{a_{n} b_{n} c_{n}}}} .
$$

Therefore,

$$
\left|\partial_{v} z_{i}\right| \geq \text { const. } \times \delta_{n}^{2 / 8-1 / 2}\left|\partial_{v} Q_{a_{n} b_{n} c_{n}}^{(i)}\right|=\text { const. } \times \delta_{n}^{-1 / 4}\left|\partial_{v} Q_{a_{n} b_{n} c_{n}}^{(i)}\right| .
$$

If we can prove that $\left|\partial_{v} Q_{a_{n} b_{n} c_{n}}^{(i)}\right|$ stays bounded away from zero as $n \rightarrow \infty$ for at least one value of $i$, we get that $\left|\partial_{v} z_{i}\right|$ diverges and we can conclude that Option 2 occurs. To control $\left|\partial_{v} Q_{a_{n} b_{n} c_{n}}^{(i)}\right|$, observe that

$$
\begin{aligned}
& \nabla Q_{a_{n} b_{n} c_{n}}^{(1)}=2 \sqrt{3}(-2 x+y, x) \\
& \nabla Q_{a_{n} b_{n} c_{n}}^{(2)}=2 \sqrt{3}(y,-2 y+x) \\
& \nabla Q_{a_{n} b_{n} c_{n}}^{(3)}=2 \sqrt{3}(-y,-x) .
\end{aligned}
$$

From these explicit formulas it is immediate to check that, whenever $(x, y) \neq$ $(0,0)$, all three gradients have non-zero norm and that there are at least two of them that are not colinear ${ }^{4}$ As a consequence (recalling that $\left(x_{n}, y_{n}\right)$ is bounded away from $(0,0))$, for any given direction $v$ one has that $\left|\partial_{v} Q_{a_{n} b_{n} c_{n}}^{(i)}\right|$ is bounded away from 0 for at least one value of $i$, as we wished to show.

\footnotetext{
${ }^{4}$ Just compute $\nabla Q_{a_{n} b_{n} c_{n}}^{(i)} \cdot\left[\nabla Q_{a_{n} b_{n} c_{n}}^{(j)}\right]^{\perp}$ for all $i \neq j$, with $v^{\perp}$ the vector $v$ rotated by $\pi / 2$, and check that only for $(x, y)=0$ the three products vanish simultaneously.
} 
Proof of Theorem 4.4. Point (I): $f$ is surjective $(f(W)=\mathcal{A})$. Fix some $\bar{w} \in W$ and let $\bar{z}=f(\bar{w})$. We define on $\mathcal{A}$ the radial vector field $v(z)=\bar{z}-z$ that points everywhere towards $\bar{z}$. Given $z \in \mathcal{A}$, we let for $t \geq 0$

$$
y^{z}(t)=\bar{z}+e^{-t}(z-\bar{z}),
$$

that solves

$$
\frac{d}{d t} y^{z}(t)=v\left(y^{z}(t)\right), \quad y^{z}(0)=z
$$

and note that $\lim _{t \rightarrow \infty} y^{z}(t)=\bar{z}$. Thanks to Theorem $4.2, \bar{z}$ is in the interior of $f(W)$, so there exists $0 \leq \tau^{z}<\infty$ such that $y^{z}\left(\tau^{z}\right) \in f(W)$. Let $w_{\tau^{z}} \in W$ be such that $f\left(w_{\tau^{z}}\right)=y^{z}\left(\tau^{z}\right)$. We let $w^{z}(t)$ be the solution of the differential equation on $W$

$$
\left\{\begin{array}{l}
\frac{d}{d t} w^{z}(t)=V\left(w^{z}(t)\right) \cdot v\left(f\left(w^{z}(t)\right)\right) \\
w^{z}\left(\tau^{z}\right)=w_{\tau^{z}}
\end{array}\right.
$$

where

$$
V(w)=[D f(w)]^{-1} .
$$

The existence of the inverse of the matrix $D f(w)$ is guaranteed by Theorem 4.2. The solution $w^{z}(t)$ exists at least locally around $t=\tau^{z}$.

Let $I$ be the interval of definition of the solution, and $\underline{t}=\inf \{s: s \in I\}$. For every $t \in \mathbb{R}_{+} \cap I$ we have $f\left(w^{z}(t)\right)=y^{z}(t)$ since, as one easily checks, both quantities verify the same differential equation and take the same value for $t=\tau^{z}$. We wish to show that $\underline{t}<0$, so that $f\left(w^{z}(0)\right)=z$, i.e. $z \in f(W)$ and in turn (by the arbitrariness of $z$ ) this implies $f(W)=\mathcal{A}$.

Let us assume by contradiction that $\underline{t} \geq 0$. Recalling Remark 4.3 and Theorem 4.2, we have that $D f(w)$ is $C^{\infty}$ (actually analytic) in $W$ and $\operatorname{det}(D f(w))$ is bounded away from zero in compact subsets of $W$. Therefore, the vector field $V(w) \cdot v(f(w))=[D f(w)]^{-1} \cdot v(f(w))$ is $C^{\infty}$ and bounded, away from the boundary of $W$. As a consequence, we have that there exists a sequence $s_{n} \searrow \underline{t}$ such that $w^{z}\left(s_{n}\right)$ approaches $\partial W$ as $n \rightarrow \infty$ (otherwise the solution could be extended to short times before $\underline{t})$. By Proposition 4.5 , one deduces that the sequence $f\left(w^{z}\left(s_{n}\right)\right)=y^{z}\left(s_{n}\right)$ cannot have a limit in $\mathcal{A}$. However, from $(39)$ such limit exists and is simply $\bar{z}+e^{-t}(z-\bar{z})$, which belongs to $\mathcal{A}$ (recall that $z, \bar{z}$ are in $\mathcal{A}$, remark that $\mathcal{A}$ is convex and that $\bar{z}+e^{-t}(z-\bar{z})$ is a convex combination of $z$ and $\left.\bar{z}\right)$.

Point (II): $f$ is bijective and a diffeomorphism. We know from point (I) that $f$ is surjective, and from Theorem 4.2 that it is a local diffeomorphism. It remains only to prove that $f^{-1}(\bar{z})$ is uniquely defined for every $\bar{z} \in \mathcal{A}$ (injectivity). This is essentially identical to the proof of injectivity in Hadamard's theorem (cf. [8, Theorem A]), so we will just sketch the main steps.

First, the set $f^{-1}(\bar{z})=\{w \in W: f(w)=\bar{z}\}$ is finite: otherwise, by Proposition 4.5 (compact sets have compact pre-images) it would contain an accumulation point $w_{\infty}$ in $W$. This would contradict Theorem 4.2, since the determinant of $D f\left(w_{\infty}\right)$ is non-zero, so that $f$ is locally one-to-one in a neighborhood of $w_{\infty}$.

Second, to each $w_{i} \in f^{-1}(\bar{z})$ is associated the set

$$
W_{i}=\left\{w_{0} \in W: \lim _{t \rightarrow \infty} w(t)=w_{i}\right\},
$$


with $w(t)$ the solution of the Cauchy problem

$$
\left\{\begin{array}{l}
\frac{d}{d t} w(t)=V(w(t)) \cdot v(f(w(t))) \\
w(0)=w_{0}
\end{array}\right.
$$

Recall that $f(w(t))=y^{z_{0}}(t)$, with $y^{z_{0}}(t)$ defined in (39) and $z_{0}=f\left(w_{0}\right)$. Since $y^{z_{0}}(t)$ stays in a compact set uniformly for $t \geq 0$, using again Proposition 4.5 we see that $w(t)$ exists for all positive times (it never approaches the boundary of $W)$. But $y^{z_{0}}(t)$ converges to $\bar{z}$ as $t \rightarrow \infty$, so that $w(t)$ tends to an inverse of $\bar{z}$ : thanks to the arbitrariness of $w_{0}$, this implies that $W=\cup_{i} W_{i}$. Moreover, each $W_{i}$ is open, by continuity of solutions of (42) with respect to initial conditions. Given that the $W_{i}$ are disjoint and that $W$ is open and connected, one deduces that $f^{-1}(\bar{z})$ contains a single element.

\section{Monotonicity And CONSTRAined DYNAMiCS}

As well as in previous works on lozenge dynamics [25, 1, 2, 17, monotonicity will play an important role. Let us briefly recall the basic idea.

In the set of stepped monotone interfaces we introduce a partial order where $h \leq h^{\prime}$ if $h(v) \leq h^{\prime}(v)$ for every $v$. It is well known that dynamics conserves the partial order: give a discrete domain $U_{L}$, it is possible to couple in the same probability space all the evolutions $h_{t}^{\eta ; \varphi_{L}}$ with boundary height $\varphi_{L}$ and initial condition $\eta \in \Omega_{U_{L}, \varphi_{L}}$ in such a way that, $\mathbb{P}$-almost surely,

$$
h_{t}^{\eta, \varphi_{L}} \leq h_{t}^{\eta^{\prime}, \varphi_{L}^{\prime}} \quad \text { for every } \quad t \geq 0, \quad \text { if } \eta \leq \eta^{\prime} \text { and } \varphi_{L} \leq \varphi_{L}^{\prime} .
$$

An immediate consequence on the equilibrium measures is that $\pi_{U_{L}}^{\varphi_{L}}$ is stochastically dominated by $\pi_{U_{L}}^{\varphi_{L}^{\prime}}$.

Consider two stepped monotone surfaces with height functions $h^{-}, h^{+}$such that $h^{-} \leq h^{+}$. Let moreover $U_{L}$ be a discrete domain and $\varphi_{L}$ be a boundary height such that $h^{-} \leq \varphi_{L} \leq h^{+}$on $U^{e x t}$. The dynamics in $U_{L}$ with boundary height $\varphi_{L}$, "ceiling" $h^{+}$and "floor" $h^{-}$is defined as the usual dynamics $h_{t}$, except that any updates that would lead to a violation of the inequalities

$$
h^{-} \leq h_{t} \leq h^{+}
$$

are discarded (censored). Of course, we will assume that the initial condition $\eta$ does satisfy $h^{-} \leq \eta \leq h^{+}$. The invariant measure of the constrained dynamics is simply the uniform measure $\pi_{U_{L}}^{\varphi_{L}}$ conditioned on the interface being between floor and ceiling, i.e.

$$
\pi_{U_{L}}^{\varphi_{L}}\left(\cdot \mid h^{-} \leq \cdot \leq h^{+}\right) .
$$

Define the distance between floor and ceiling as

$$
\max _{v \in U_{L}}\left(h^{+}(v)-h^{-}(v)\right) .
$$

Then:

Lemma 5.1. [1, Theorem 4.3] The Glauber dynamics in a discrete domain $U_{L}$ of diameter $D$ in the graph-distance, with floor and ceiling at distance $H / L$, has $T_{\text {mix }}=O\left(D^{2} H^{2}(\log D)^{2}\right)$. 
Take $U_{L}$ to be the discretization of a domain $U$, so that its graph-distance diameter $D$ is of order $L$. Note that, if we let $h^{ \pm}=\varphi_{L} \pm A$ with $A=O(1)$ sufficiently large then the constrained dynamics exactly coincides with the unconstrained one. This is simply because the height functions $h$ and $\varphi_{L}$ change by $\pm 1 / L$ or 0 along edges of $\mathcal{T}_{L}$ : if $u \in \partial U_{L}$ and $v \in U^{i n t}$ and $u, v$ are at graph-distance $d(u, v)$, one has

$$
h(v) \leq h(u)+d(u, v) / L
$$

and

$$
h^{+}(v) \geq h^{+}(u)-d(u, v) / L=\varphi_{L}(u)+A-d(u, v) / L .
$$

Given that $h(u)=\varphi_{L}(u)$ on $\partial U_{L}$, we see that

$$
h^{+}(v) \geq h(u)+A-d(u, v) / L \geq h(v)+A-2 d(u, v) / L .
$$

If $A$ is chosen larger than $2 D / L=O(1)$ we have then $h(v) \leq h^{+}(v)$ (and analogously $\left.h(v) \geq h^{-}(v)\right)$ deterministically. Hence the floor/ceiling constraints are automatically satisfied by the unconstrained dynamics. On the other hand, if $h^{ \pm}=\varphi_{L} \pm A$ then the distance between floor and ceiling is $2 A$. Therefore, an immediate consequence of Lemma 5.1 is:

Corollary 5.2. Let the discrete domain $U_{L}$ be a discretization of a domain $U$. For any boundary height $\varphi_{L}$, the mixing time of the Glauber dynamics with neither floor nor ceiling is smaller than $C L^{4}(\log L)^{2}$ for some constant $C$ depending only on $U$.

\section{Proof of Theorem 3.1}

Here we make a few comments about the idea of the proof and its structure. Recall from the introduction that we want to show that the interface stays with very high probability "trapped" between two deterministic surfaces that evolve on a time scale just slower than diffusive and both tend to the macroscopic shape. We will only consider the upper bound in the following because the proof of the lower bound is identical.

The first step, that does not require much work, is to realize that it is enough to prove that when the initial condition is at distance $2 \epsilon_{L}$ from equilibrium (for some suitably small $\epsilon_{L}$ ) then within time $L^{2+o(1)}$ the interface reaches distance $\epsilon_{L}$ (see Claim 6.1). To prove this, the key point is Claim 6.3 . that says that the height function stays with high probability below the deterministically evolving interface

$$
\tilde{\phi}_{t}:=\bar{\phi}+\epsilon_{L}\left(1-t / L^{2+o(1)}\right) \psi,
$$

with a well chosen function $\psi \geq 2$, until the time when $\left(1-t / L^{2+o(1)}\right)$ becomes sufficiently small. As mentioned in the introduction, we prove the bound by looking at "mesoscopic" regions of size $L^{-1 / 2+o(1)}$ and at time increments $L^{1+o(1)}$, that are small with respect to the diffusive scaling. The choice of $\psi$ will be justified in Remark 6.4. In practice, one must guarantee that $\hat{\mathcal{L}} \psi$ (with $\hat{\mathcal{L}}$ the linearization of the elliptic operator in (4), that should determine the interface drift, see (1)) is comparable with $\Delta \psi$ (with $\Delta$ the usual Laplacian).

For simplicity we will write $\pi_{L}$ for the equilibrium measure $\pi_{U_{L}}^{\varphi_{L}}$ and as usual $\bar{\phi}$ denotes the macroscopic shape in $U$ with boundary height $\varphi$. 
Let $\epsilon_{L}=1 / \log L$. To prove Theorem 3.1 it is sufficient to prove that

$$
\sup _{t>c(\delta) L^{2+\delta}} \mathbb{P}\left(\exists v \in U_{L}: h_{t}(v)-\bar{\phi}(v)>2 \epsilon_{L}\right)=o(1)
$$

and

$$
\sup _{t>c(\delta) L^{2+\delta}} \mathbb{P}\left(\exists v \in U_{L}: h_{t}(v)-\bar{\phi}(v)<-2 \epsilon_{L}\right)=o(1),
$$

with bounds uniform in the initial condition $\eta$ (we omit for lightness the argument $\eta$ in $h_{t}^{\eta}$ ). We will prove only (44), the proof of (45) being essentially identical.

Let $G$ be a positive constant, independent of $\eta$ and $L$, that will be fixed in a moment (it will depend only on the diameter of $U$ ). We have:

Claim 6.1. For $i=0,1, \ldots,\left\lfloor G / \epsilon_{L}\right\rfloor-1$,

$$
\mathbb{P}\left(h_{t} \leq G-i \epsilon_{L}+\bar{\phi} \text { for every } t \in\left[T_{i}, L^{5}\right]\right) \geq 1-i / L
$$

where

$$
T_{i}=L^{2+\delta / 2} i
$$

When we write $h_{t} \leq g$ like in (46), what we mean exactly is that for every $u \in U_{L} \cap \mathcal{T}_{L}$ one has $h_{t}(u) \leq g(u)$.

Proof of (44) given Claim 6.1. For $i=\left\lfloor G / \epsilon_{L}\right\rfloor-1$ we have

$$
T_{i}=O\left(L^{2+\delta / 2} / \epsilon_{L}\right)=O\left(L^{2+\delta / 2} \log L\right) \ll c(\delta) L^{2+\delta}
$$

and we obtain that

$$
\mathbb{P}\left(h_{t} \leq \bar{\phi}+2 \epsilon_{L} \quad \text { for every } t \in\left[c(\delta) L^{2+\delta}, L^{5}\right]\right)=1+o(1) .
$$

On the other hand, we know from Corollary 5.2 that the mixing time of the dynamics is $T_{\text {mix }}=O\left(L^{4}(\log L)^{2}\right)$. Therefore, from (10) we see that for times larger than $L^{5}$ the system is at equilibrium (modulo a negligible error term $O\left(\exp \left(-L /(\log L)^{2}\right)\right)$, uniform in time and in $\left.\eta\right)$ and we deduce that

$$
\pi_{L}\left(h \leq \bar{\phi}+2 \epsilon_{L}\right)=1+o(1)
$$

so that

$$
\sup _{t>L^{5}} \mathbb{P}\left(h_{t} \leq \bar{\phi}+2 \epsilon_{L}\right)=\pi_{L}\left(h \leq \bar{\phi}+2 \epsilon_{L}\right)+O\left(\exp \left(-L /(\log L)^{2}\right)\right)=1+o(1) .(49
$$

Equations (47) and (49) imply (44). It will be clear from the proof of Claim 6.1 that in 446) we could have replaced $L^{5}$ with any other larger power of $L$.

Proof of Claim 6.1. We prove (46) by induction on $i$. The functions $h_{t}$ and $\bar{\phi}$ are uniformly 1-Lipschitz in space and they coincide on the boundary of $U_{L}$ : therefore, Eq. (46) for $i=0$ is trivially true (for every $t \geq 0$ ) if $G$ is chosen large enough depending on the diameter of $U$.

Definition 6.2. Set for $(x, y) \in \mathbb{R}^{2}$

$$
\psi(x, y)=\psi(0,0)-e^{x / \xi}-e^{y / \xi}
$$


with $\xi \ll 1$ but independent of $L$ and the constant $\psi(0,0)$ chosen so that $\inf \{\psi(x, y),(x, y) \in U\}=2$. Let $\psi_{\max }=\max \{\psi(x, y):(x, y) \in U\}$ and $N \sim L\left(1-1 / \psi_{\max }\right)$ the smallest integer such that

$$
\left(1-\frac{N}{L}\right) \psi_{\max } \leq 1
$$

To prove 46 for $i+1$ given the same statement for $i$, we proceed as follows. Since $\psi \geq 2$ in $U$, the inductive hypothesis (i.e. (46) for $i$ ) implies

$$
\mathbb{P}\left(h_{t} \leq G-(i+2) \epsilon_{L}+\bar{\phi}+\epsilon_{L} \psi \text {, for every } T_{i} \leq t \leq L^{5}\right) \geq 1-i / L .
$$

Define

$$
\gamma_{i, j}=G-(i+2) \epsilon_{L}+\bar{\phi}+\epsilon_{L}(1-j / L) \psi,
$$

let $T_{i, j}=T_{i}+j L^{1+\delta / 4}$ and $E_{i, j}$ be the event

$$
E_{i, j}=\left\{h_{t} \leq \gamma_{i, j} \text { for every } T_{i, j} \leq t \leq L^{5}\right\} .
$$

We will prove:

Claim 6.3. Fix $0 \leq i<\left\lfloor G / \epsilon_{L}\right\rfloor-1$ and assume that (46) holds. For $j \leq N$,

$$
\mathbb{P}\left(E_{i, j}\right) \geq 1-i / L-j / L^{3} .
$$

Taking $j=N$, we obtain the claim (46) for $i+1$, since $T_{i, N} \leq T_{i+1}$,

$$
\gamma_{i, N} \leq G-(i+1) \epsilon_{L}+\bar{\phi}
$$

and

$$
1-i / L-N / L^{3} \geq 1-(i+1) / L .
$$

This concludes the proof of Claim 6.1, assuming Claim 6.3 .

Remark 6.4. The choice of $\psi$, which might look at first sight rather arbitrary, is dictated by the following reasoning. At time $T_{i}$ we have $h_{t} \leq$ $\bar{\phi}+\epsilon_{L} \psi+c$, with $c$ the constant $G-(i+2) \epsilon_{L}$. From the discussion in the Introduction, we expect the macroscopic evolution of the interface under diffusive time scaling to be given by (1). Linearizing the differential operator $\mathcal{L}$ around $\bar{\phi}+c$ and observing that $\mathcal{L}(\bar{\phi}+c)=0$, we find that

$$
\mathcal{L}\left(\bar{\phi}+c+\epsilon_{L} \psi\right)=\epsilon_{L} \hat{\mathcal{L}} \psi+O\left(\epsilon_{L}^{2}\right)
$$

with $\hat{\mathcal{L}}$ the linear elliptic operator

$$
\begin{aligned}
\hat{\mathcal{L}} \psi=\sum_{i, j=1}^{2} & a_{i j}(\nabla \bar{\phi}) \partial_{i j}^{2} \psi \\
& +\sum_{i, j=1}^{2}\left[\left.\left(\partial_{x} \psi\right) \partial_{s} a_{i j}(s, t)\right|_{(s, t)=\nabla \bar{\phi}}+\left.\left(\partial_{y} \psi\right) \partial_{t} a_{i j}(s, t)\right|_{(s, t)=\nabla \bar{\phi}}\right] .
\end{aligned}
$$

Now observe that the Hessian matrix of our $\psi$ is diagonal, with negative diagonal entries:

$$
H^{\psi}=-\frac{1}{\xi^{2}}\left(\begin{array}{cc}
e^{x / \xi} & 0 \\
0 & e^{y / \xi}
\end{array}\right) .
$$

Therefore, the first sum in (55) is strictly and pointwise negative, uniformly in $U$ (the diagonal elements $a_{i i}$ are positive) and the second sum can be 
neglected, if $\xi$ is small (because $\left|\partial_{x} \psi\right| \ll\left|\partial_{x}^{2} \psi\right|$ and similarly for the $y$ derivatives). In conclusion, with our choice of $\psi, \mathcal{L}\left(\bar{\phi}+c+\epsilon_{L} \psi\right)$ is everywhere negative, so the interface feels a negative drift that pushes it towards the equilibrium shape. The drift is of order $-L^{-2}$ (recall that, in (1), $\tau$ is the rescaled time $\left.\tau=t / L^{2}\right)$. This heuristic reasoning is what is behind Claim 6.3. Indeed, going from $j$ to $j+1$ corresponds to lowering the interface by $\approx 1 / L$, in a time $T_{i, j+1}-T_{i} \approx L$, i.e. corresponds to a negative drift of order $-1 / L^{2}$.

Proof of Claim 6.3. We proceed by induction on $j$ and we observe that for $j=0$ the claim is trivial (it just reduces to (51), that is a consequence of (46) which we assumed to hold for the value $i$ ). We want to prove (54), given the same claim for $j-1$.

Let $V$ be a shrinking of $U$ by $\epsilon_{L}^{2}$, i.e. let

$$
V=U \backslash \cup_{x \in \partial U} B\left(x, \epsilon_{L}^{2}\right),
$$

with $B(x, r)$ the ball of radius $r$ centered at $x$.

Remark 6.5. We claim first of all that it is sufficient to prove (46) at lattice sites $v \in V$. Indeed, recall that the height on $\partial U_{L}$ is always fixed (for all times) to the boundary height $\varphi_{L}$. Since both the height function $h_{t}$ and $\bar{\phi}$ are uniformly 1-Lipschitz in space and $\left|h_{t}-\bar{\phi}\right| \simeq\left|\varphi_{L}-\varphi\right|=O(1 / L)$ at the boundary $\partial U_{L}$ one deduces that, deterministically, $\left|h_{t}-\bar{\phi}\right|=O\left(\epsilon_{L}^{2}\right)$ in $U_{L} \backslash V$. On the other hand, for $i<\left\lfloor G / \epsilon_{L}\right\rfloor-1$ and $j \leq N$

$$
\begin{aligned}
\gamma_{i, j}-h_{t}=\bar{\phi}-h_{t} & +G-(i+2) \epsilon_{L}+(1-j / L) \epsilon_{L} \psi \\
& \geq \bar{\phi}-h_{t}+\epsilon_{L}(1-N / L) \psi_{\max } \frac{\psi}{\psi_{\max }} \geq \bar{\phi}-h_{t}+\epsilon_{L} / \psi_{\max }
\end{aligned}
$$

since $\psi \geq 2$ and $(1-N / L) \psi_{\max }=1+o(1)$. We have seen that for the sites within distance $\epsilon_{L}^{2}$ from $\partial U$ one has $\left|\bar{\phi}-h_{t}\right|=O\left(\epsilon_{L}^{2}\right) \ll \epsilon_{L} / \psi_{\text {max }}$ deterministically, so the inequality $h_{t} \leq \gamma_{i, j}$ holds automatically.

In conclusion, we do not have to worry about lattice sites too close to the boundary $\partial U$, see also Remark 6.6

One has

$$
\mathbb{P}\left(E_{i, j}\right) \geq \mathbb{P}\left(E_{i, j-1}\right)-\mathbb{P}\left(E_{i, j-1} ; E_{i, j}^{c}\right) \geq 1-\frac{i}{L}-\frac{j-1}{L^{3}}-\mathbb{P}\left(E_{i, j-1} ; E_{i, j}^{c}\right) .
$$

Next, via a union bound,

$$
\mathbb{P}\left(E_{i, j-1} ; E_{i, j}^{c}\right) \leq \sum_{u \in V} \mathbb{P}\left(E_{i, j-1} ; h_{t}(u)>\gamma_{i, j}(u) \text { for some } t \in\left[T_{i, j}, L^{5}\right]\right) .
$$

Since there are $O\left(L^{2}\right)$ sites $u \in V$, it is sufficient to prove

$$
\mathbb{P}\left(E_{i, j-1} ; h_{t}(u)>\gamma_{i, j}(u) \text { for some } t \in\left[T_{i, j}, L^{5}\right]\right) \leq 1 / L^{6}
$$

to deduce (54). that

Let the stepped monotone interface $\gamma_{i, j-1}^{(L)}$ be a discretization of $\gamma_{i, j-1}$ such

$$
\gamma_{i, j-1}^{(L)}>\gamma_{i, j-1}
$$

(strict inequality) and $\left\{\hat{h}_{t}\right\}_{t \geq T_{i, j-1}}$ be the Markov dynamics with (random) initial condition $h_{T_{i, j-1}}$ at time $T_{i, j-1}$, and such that: 
- if $h_{T_{i, j-1}} \leq \gamma_{i, j-1}^{(L)}$, then $\hat{h}_{t}$ is the dynamics with ceiling $\gamma_{i, j-1}^{(L)}$;

- if instead $h_{T_{i, j-1}} \nless \gamma_{i, j-1}^{(L)}$, then $\hat{h}_{t}=h_{T_{i, j-1}}$ for every $t \geq T_{i, j-1}$.

Note that, on the event $E_{i, j-1}$, one has $h_{T_{i, j-1}} \leq \gamma_{i, j-1}^{(L)}$ and moreover the two dynamics $h_{t}$ and $\hat{h}_{t}$ can be coupled so that they exactly coincide in the time interval $\left[T_{i, j-1}, L^{5}\right]$. In fact, from the definition of dynamics with ceiling (Section 5) the two dynamics coincide until the first time $\tau$ when $h_{\tau}(v)=\gamma_{i, j-1}^{(L)}(v)$ for some $v$; on the event $E_{i, j-1}$ one has $h_{t} \leq \gamma_{i, j-1}<\gamma_{i, j-1}^{(L)}$ up to time $L^{5}$ time and therefore $\tau \geq L^{5}$. Therefore, the probability in (61) can be upper bounded by

$$
\mathbb{P}\left(\hat{h}_{t}(u)>\gamma_{i, j}(u) \text { for some } t \in\left[T_{i, j}, L^{5}\right] \mid \hat{h}_{T_{i, j-1}}=h_{T_{i, j-1}} \leq \gamma_{i, j-1}^{(L)}\right) .
$$

Next, we want to reduce from the dynamics $\hat{h}_{t}$ in the whole $U_{L}$ to a dynamics where only the height function in a much smaller domain $D_{u}$ evolves. Given a lattice site $u \in V$ let $D_{u}$ be a disk 5 of radius $L^{-1 / 2+\delta / 100}$ centered at $u$ : from the definition (57) of $V$, we see that the disk $D_{u}$ is entirely contained in $U$, since $L^{-1 / 2+\delta / 100} \ll \epsilon_{L}^{2}$.

We start by observing that, by monotonicity, since we want to upper bound (63), we are allowed to change the random configuration $\hat{h}_{T_{i, j-1}}$ at time $T_{i, j-1}$ to the deterministic configuration $\gamma_{i, j-1}^{(L)} \geq \hat{h}_{T_{i, j-1}}$, and to freeze $\hat{h}_{t}(v)$ to $\gamma_{i, j-1}^{(L)}(v)$ for times $t \geq T_{i, j-1}$ and sites $v$ outside $D_{u}$. In words, we are pinning the height function to the ceiling outside $D_{u}$. Again by monotonicity, we impose that the evolution $\hat{h}_{t}$ has a "floor" constraint $\hat{h}_{t}(v) \geq \gamma_{i, j-1}^{(L)}-L^{-1+\delta / 40}$. We still call $\left\{\hat{h}_{t}\right\}_{t \geq T_{i, j-1}}$ the dynamics after these two modifications, and we denote $\hat{\pi}_{i, j-1}$ its equilibrium measure (it is the uniform measure on stepped monotone interfaces in $D_{u}$, with boundary height $\left.\gamma_{i, j-1}^{(L)}\right|_{\partial D_{u}}$, ceiling $\gamma_{i, j-1}^{(L)}$ and floor $\left.\gamma_{i, j-1}^{(L)}-L^{-1+\delta / 40}\right)$.

Remark 6.6. We have used crucially that $D_{u} \subset U$, more precisely that boundary sites on $\partial D_{u}$ are in $U_{L}$ to say that, on the event $E_{i, j-1}, h_{t}(v)<$ $\gamma_{i, j-1}^{(L)}(v)$ for $v \in \partial D_{u}$ and $t \in\left[T_{i, j-1}, L^{5}\right]$. If we had to consider points much closer to the boundary, we would have to take a disk $D_{u}$ of smaller diameter (so that it fits in $U_{L}$ ) and then the proof of Proposition 6.7 below would fail.

Let us assume for the moment the following equilibrium estimate:

Proposition 6.7. Let $j \leq N$ and $\hat{\pi}_{i, j-1}$ be as above. If $\xi$ in (50) is smaller than some $\xi_{0}>0$ (that is independent of $i, j, L$ ) then

$$
\hat{\pi}_{i, j-1}\left[h(u)>\gamma_{i, j-1}(u)-L^{-1+\delta / 60}\right] \leq L^{-20} .
$$

Let us conclude the proof of the step $j-1 \rightarrow j$, given Proposition 6.7. By Lemma 5.1, since the graph-distance diameter of $D_{u}$ is $O\left(L^{1 / 2+\delta / 100}\right)$ and the distance between floor $\gamma_{i, j-1}^{(L)}-L^{-1+\delta / 40}$ and ceiling $\gamma_{i, j-1}^{(L)}$ is $L^{-1+\delta / 40}$, the

\footnotetext{
5 To be precise, $D_{u}$ should be a discrete domain; take $D_{u}$ as the union of triangles in $\mathcal{T}_{L}$ contained in such a disk. For lightness of exposition, we will overlook this minor detail and just call $D_{u}$ a "disk".
} 
mixing time of the dynamics $\hat{h}_{t}$ is

$$
O\left(L^{2(1 / 2+\delta / 100)} L^{2 \delta / 40}(\log L)^{2}\right) \leq L^{1+\delta / 8} \ll T_{i, j}-T_{i, j-1}=L^{1+\delta / 4} .
$$

Therefore, at time $T_{i, j}$ equilibrium $\hat{\pi}_{i, j-1}$ has been reached, up to a negligible variation distance error $O\left(\exp \left(-L^{\delta / 8}\right)\right)$. As a consequence,

$$
\begin{array}{r}
\mathbb{P}\left(\hat{h}_{t}(u)>\gamma_{i, j}(u) \text { for some } t \in\left[T_{i, j}, L^{5}\right]\right) \\
=\mathbb{P}^{\hat{\pi}_{i, j-1}}\left(\hat{h}_{t}(u)>\gamma_{i, j}(u) \text { for some } t \in\left[T_{i, j}, L^{5}\right]\right)+O\left(\exp \left(-L^{\delta / 8}\right)\right) \\
\leq L^{7} \hat{\pi}_{i, j-1}\left(h(u)>\gamma_{i, j}(u)\right)+O\left(\exp \left(-L^{\delta / 8}\right)\right) .
\end{array}
$$

In the second line, $\mathbb{P}^{\hat{x}_{i, j-1}}$ denotes the law of the modified dynamics, with initial condition sampled from the equilibrium distribution $\hat{\pi}_{i, j-1}$; in the third line we used the standard fact that, for a continuous-time homogeneous Markov chain $X_{t}$ with invariant measure $\pi$ and any event $A$,

$$
\mathbb{P}^{\pi}\left(\exists t \in[a, b]: X_{t} \in A\right) \leq|b-a| M \pi(X \in A)
$$

with $M$ the average number of updates per unit time. In our case $M$ can be bounded by the number of lattice sites in $D_{u}$ (since each site has a mean-one Poisson clock), which is much smaller than $L^{2}$.

Note that

$$
\gamma_{i, j-1}(u)-L^{-1+\delta / 60}<\gamma_{i, j}(u):
$$

just recall (52) and observe that $L^{-1+\delta / 60}>\epsilon_{L} \psi(u) / L$. As a consequence, from Proposition 6.7

$$
\hat{\pi}_{i, j-1}\left(h(u)>\gamma_{i, j}(u)\right) \leq \hat{\pi}_{i, j-1}\left(h(u)>\gamma_{i, j-1}(u)-L^{-1+\delta / 60}\right) \leq L^{-20}
$$

and (61) follows from (63) and (65). The proof of Claim 6.3 is concluded.

Remark 6.8. The common points with the proof of [1, Theorem 2] (that is the analog of Theorems 3.1 and 3.6 in the particular case of flat macroscopic shape) are the pervasive use of monotonicity and the idea of employing Lemma 5.1 on mesoscopic domains of size slightly larger than $L^{-1 / 2}$ (specifically, $L^{-1 / 2+\delta / 100}$ here).

\section{Proof of Proposition 6.7}

Since $i$ and $j$ are fixed in this section, we write for simplicity of notation

$$
\gamma=\gamma_{i, j-1}=G-(i+2) \epsilon_{L}+\bar{\phi}+\kappa \epsilon_{L} \psi
$$

with $\kappa=(1-(j-1) / L)$. Recall that $(j-1)<N$, with $N$ as in Definition 6.2 , so that

$$
\kappa \in\left[(1+O(1 / L)) / \psi_{\max }, 1\right] .
$$

In fact, we will need that $\kappa$ is bounded away from zero uniformly in $L, i, j$.

Recall that $u=(x, y) \in V, D_{u} \subset U$ is a disk centered at $u$, of radius $L^{-1 / 2+\delta / 100}$ and that $\hat{\pi}:=\hat{\pi}_{i, j-1}$ is the uniform measure over stepped interfaces in $D_{u}$, with boundary condition $\left.\gamma_{i, j-1}^{(L)}\right|_{\partial D_{u}}$, ceiling $\gamma_{i, j-1}^{(L)}$ and floor $\gamma_{i, j-1}^{(L)}-L^{-1+\delta / 40}$. Since we want to prove the upper bound (64), we can by monotonicity remove the ceiling.

The floor cannot be removed by monotonicity. However: 
Lemma 7.1. Let $\tilde{\pi}$ be obtained from $\hat{\pi}$ by eliminating the floor constraint $h \geq \gamma_{i, j-1}^{(L)}-L^{-1+\delta / 40}$. We have $\|\hat{\pi}-\tilde{\pi}\|=O\left(L^{-n}\right)$ for any given $n$.

This will be proven at the end of this section. It is clear that it is sufficient to prove Proposition 6.7 with $\hat{\pi}$ replaced by $\tilde{\pi}$.

Call $\nabla \bar{\phi}(u)=\left(\partial_{x} \bar{\phi}(u), \partial_{y} \bar{\phi}(u)\right)$ the slope of $\bar{\phi}$ at $u$ and $H^{\bar{\phi}}(u)$ its $2 \times 2$ Hessian matrix. These are well-defined objects, since $\bar{\phi}$ is non-extremal and therefore infinitely differentiable, see Section 2.2. Similarly, call

$$
\nabla \gamma(u)=\left(\partial_{x} \gamma(u), \partial_{y} \gamma(u)\right)=\nabla \bar{\phi}(u)+\kappa \epsilon_{L} \nabla \psi(u)
$$

and

$$
H^{\gamma}(u)=H^{\bar{\phi}}(u)+\kappa \epsilon_{L} H^{\psi}(u)
$$

the slope and Hessian of $\gamma$. The argument $u$ will be omitted unless needed for clarity. Note that $\nabla \gamma$ and $H^{\gamma}$ do not in general satisfy (4), i.e. in general

$$
\mathbf{a}(\nabla \gamma) \cdot H^{\gamma}:=\sum_{i, j=1,2} a_{i j}(\nabla \gamma) H_{i j}^{\gamma} \neq 0,
$$

because $\gamma$ is not the equilibrium shape with some boundary height. In other words, $\left(\nabla \gamma, H^{\gamma}\right)$ is not the local structure of any macroscopic shape.

Remark 7.2. One has

$$
\nabla \psi=-\frac{1}{\xi}\left(e^{x / \xi}, e^{y / \xi}\right)
$$

and

$$
\|\nabla \gamma-\nabla \bar{\phi}\|=\kappa \epsilon_{L}\|\nabla \psi\| \leq \mathcal{K}\left(\xi, \kappa, \epsilon_{L}, u\right):=\sqrt{2} \frac{\kappa \epsilon_{L}}{\xi} e^{\max (x, y) / \xi},
$$

which is o(1) when $L \rightarrow \infty$. In particular, since $\nabla \bar{\phi}$ is uniformly bounded away from $\partial \mathbb{T}$, so is $\nabla \gamma$.

Define then the $2 \times 2$ matrix

$$
\bar{H}=H^{\bar{\phi}}-\frac{H^{\bar{\phi}} \cdot \mathbf{a}(\nabla \gamma)}{H^{\psi} \cdot \mathbf{a}(\nabla \gamma)} H^{\psi}
$$

with $H^{\psi}$ defined in (56). The denominator is non-zero always: recall from Section 2.2 that the diagonal elements $a_{i i}$ given in (5) are positive away from $\partial \mathbb{T}$ and that $H^{\psi}$ is diagonal, with negative diagonal entries, see (56). Remark also that, in contrast with 69,

$$
\mathbf{a}(\nabla \gamma) \cdot \bar{H}=0 .
$$

In other words, we have added the right correction to $H^{\gamma}$ so that $(\nabla \gamma, \bar{H})$ is a local structure of a macroscopic shape. We further remark that

$$
H^{\gamma}-\bar{H}=\left(\kappa \epsilon_{L}+\frac{H^{\bar{\phi}} \cdot \mathbf{a}(\nabla \gamma)}{H^{\psi} \cdot \mathbf{a}(\nabla \gamma)}\right) H^{\psi}=\kappa \epsilon_{L}(1+O(\xi)) H^{\psi} .
$$

To see this, observe that for the denominator

$$
\left|H^{\psi} \cdot \mathbf{a}(\nabla \gamma)\right| \geq \frac{c}{\xi^{2}} e^{\max (x, y) / \xi}
$$


with $c=\min _{(x, y) \in U} \min \left(a_{11}(\nabla \gamma), a_{22}(\nabla \gamma)\right)>0$; for the numerator,

$$
\begin{array}{r}
H^{\bar{\phi}} \cdot \mathbf{a}(\nabla \gamma)=H^{\bar{\phi}} \cdot \mathbf{a}(\nabla \bar{\phi})+H^{\bar{\phi}} \cdot(\mathbf{a}(\nabla \gamma)-\mathbf{a}(\nabla \bar{\phi})) \\
=0+H^{\bar{\phi}} \cdot(\mathbf{a}(\nabla \gamma)-\mathbf{a}(\nabla \bar{\phi})) .
\end{array}
$$

From (71) and (75) one deduces

$$
\frac{H^{\bar{\phi}} \cdot \mathbf{a}(\nabla \gamma)}{H^{\psi} \cdot \mathbf{a}(\nabla \gamma)}=O\left(\kappa \epsilon_{L} \xi\right)
$$

and 74 follows.

Let

$$
z(u)=\left(\partial_{x} \gamma(u), \partial_{y} \gamma(u), \bar{H}_{11}(u), \bar{H}_{12}(u)\right) \in \mathcal{A}
$$

and define

$$
w(u)=(A(u), B(u), X(u), Y(u))=f^{-1}(z(u)) \in W,
$$

with $f: W \mapsto \mathcal{A}$ the function of Theorem 4.4. That is, recalling also (73), $(\nabla \gamma(u), \bar{H}(u))$ is the local structure of the macroscopic shape $\bar{\phi}_{A(u) B(u) C(u)}$ in the hexagon $\bigcirc_{A(u) B(u) C(u)}$, with $C(u)=1-A(u)-B(u)$, at the point $(X(u), Y(u)) \in \bigcirc_{A(u) B(u) C(u)}$.

Remark 7.3. Observe that, since $\bar{\phi}$ is non-extremal, the closure $K$ of the set

$$
K^{\prime}=\left\{\left(\partial_{x} \bar{\phi}(u), \partial_{y} \bar{\phi}(u), \partial_{x}^{2} \bar{\phi}(u), \partial_{x y}^{2} \bar{\phi}(u)\right), u \in U\right\}
$$

is a compact subset of the open set $\mathcal{A}$. By Proposition 4.5. $f^{-1}(K)$ is a compact subset of the open set $W$. Next, note that

$$
\sup _{u \in U}\left\|z(u)-\left(\partial_{x} \bar{\phi}(u), \partial_{y} \bar{\phi}(u), \partial_{x}^{2} \bar{\phi}(u), \partial_{x y}^{2} \bar{\phi}(u)\right)\right\|=o(1)
$$

when $L \rightarrow \infty$. Indeed, $\|\nabla \gamma-\nabla \bar{\phi}\|$ was bounded in (71), while

$$
\left\|\bar{H}-H^{\bar{\phi}}\right\|=\frac{\left|H^{\bar{\phi}} \cdot \mathbf{a}(\nabla \gamma)\right|}{\left|\left(H^{\psi} /\left\|H^{\psi}\right\|\right) \cdot \mathbf{a}(\nabla \gamma)\right|}=O\left(\sup _{u \in U} \mathcal{K}\left(\xi, \kappa, \epsilon_{L}, u\right)\right)=o(1):
$$

for the numerator see (76) and (71), while the denominator is lower bounded by

$$
(1 / \sqrt{2}) \times \min _{(x, y) \in U} \min \left(a_{11}(\nabla \gamma), a_{22}(\nabla \gamma)\right)
$$

which is positive, as discussed before.

As a consequence of (80) and of Proposition 4.5, we see that $\{w(u), u \in$ $U\}$ is contained in a compact subset of $W$. In other words, uniformly in $u \in U, A(u), B(u), C(u)$ are bounded away from zero and $(X(u), Y(u))$ is

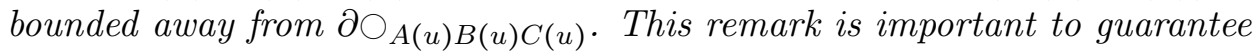
that, when one applies Theorem 3.4, estimates one obtains are uniform with respect to $u$. Uniformity will be not be recalled explicitly later.

From now on, for lightness of notation we remove the argument $u$ from $A(u), B(u), C(u)$. Call, as in Section 3.2. $\pi_{L}^{A B C}$ the uniform measure in the hexagon $\bigcirc_{A_{L} B_{L} C_{L}}$ (the discretization of $\bigcirc_{A B C}$ ) and $\bar{\phi}_{A B C}$ the macroscopic shape. Translate $\bigcirc_{A_{L} B_{L} C_{L}}$ in the $P_{111}$ plane so that $u=(x, y)$ and 
$(X(u), Y(u))$ coincide, and add a suitable global constant to the boundary height $\varphi_{a_{L} b_{L} c_{L}}$ on $\partial \bigcirc_{A_{L} B_{L} C_{L}}$, so that

$$
\pi_{L}^{A B C}(h(u))=\gamma(u) .
$$

We are at last in a position to prove the claim of Proposition 6.7, i.e. that

$$
\tilde{\pi}\left(h(u)>\gamma(u)-L^{-1+\delta / 60}\right) \leq L^{-20}
$$

(recall that $\tilde{\pi}$ differs from $\hat{\pi}$ in that the floor constraint $h \geq \gamma_{i, j-1}^{(L)}-L^{-1+\delta / 40}$ has been removed).

Recall that the boundary condition on $\partial D_{u}$ (that is a disk of radius $L^{-1 / 2+\delta / 100}$ centered at $u$ ), is $\gamma_{i, j-1}^{(L)}$, that is a discretization of $\gamma=\gamma_{i, j-1}$. We have for $v \in D_{u}$

$$
\begin{aligned}
\gamma_{i, j-1}^{(L)}(v) & =\gamma(v)+O(1 / L) \\
= & \gamma(u)+(v-u) \cdot \nabla \gamma(u)+\frac{1}{2}(v-u) \cdot H^{\gamma}(u) \cdot(v-u)+O(1 / L)
\end{aligned}
$$

where we used $\|u-v\| \sim L^{-1 / 2+\delta / 100}$ and smoothness of $\gamma$ to ignore higherorder terms in the Taylor expansion of $\gamma$ around $u$. Next, write $H^{\gamma}(u)=$ $\bar{H}(u)+\left(H^{\gamma}(u)-\bar{H}(u)\right)$, with $\bar{H}$ defined in (72). From (74) and (56) we have for $\xi$ small enough

$$
(v-u) \cdot\left(H^{\gamma}(u)-\bar{H}(u)\right) \cdot(v-u) \leq-C_{1} \epsilon_{L}\|u-v\|^{2},
$$

with $C_{1}(\xi, \kappa)$ a strictly positive constant, independent of $L$ (recall from 68 ) that $\kappa$ is bounded away from zero). Therefore,

$\gamma_{i, j-1}^{(L)}(v) \leq \gamma(u)+(v-u) \cdot \nabla \gamma(u)+\frac{1}{2}(v-u) \cdot \bar{H}(u) \cdot(v-u)-C_{1} \epsilon_{L}\|u-v\|^{2}+O(1 / L)$.

Now recall that $\nabla \gamma(u)$ and $\bar{H}(u)$ are also the gradient and Hessian at $u$ of $\bar{\phi}_{A B C}$, the macroscopic shape in the hexagon $\bigcirc_{A B C}$. A second-order Taylor expansion and $(82)$ give then

$$
\begin{aligned}
& \gamma_{i, j-1}^{(L)}(v) \leq\left(\gamma(u)-\bar{\phi}_{A B C}(u)\right)+\bar{\phi}_{A B C}(v)-C_{1} \epsilon_{L}\|u-v\|^{2}+O(1 / L) \\
& \quad=\left(\pi_{L}^{A B C}(h(u))-\bar{\phi}_{A B C}(u)\right)+\bar{\phi}_{A B C}(v)-C_{1} \epsilon_{L}\|u-v\|^{2}+O(1 / L) .
\end{aligned}
$$

Thanks to Theorem 3.4 we have $\left(\pi_{L}^{A B C}(h(u))-\bar{\phi}_{A B C}(u)\right)=\left(\pi_{L}^{A B C}(h(v))-\right.$ $\left.\bar{\phi}_{A B C}(v)\right)+O(1 / L)$ and finally

$$
\gamma_{i, j-1}^{(L)}(v) \leq \pi_{L}^{A B C}(h(v))-C_{1} \epsilon_{L}\|u-v\|^{2}+O(1 / L) .
$$

Taking $v \in \partial D_{u}$, so that $\|u-v\| \sim L^{-1 / 2+\delta / 100}$, we deduce that the boundary height $\left.\gamma_{i, j-1}^{(L)}\right|_{\partial D_{u}}$ in the measure $\tilde{\pi}$ is lower than the function

$$
\partial D_{u} \ni v \mapsto \pi_{L}^{A B C}(h(v))-C_{2} \epsilon_{L} L^{-1+\delta / 50} .
$$

An immediate application of Proposition 3.5 gives that the $\tilde{\pi}$-probability that

$$
h(u)>\gamma(u)-L^{-1+\delta / 60}=\pi_{L}^{A B C}(h(u))-L^{-1+\delta / 60}
$$

is $O\left(L^{-n}\right)$ for any given $n$. Choosing $n>20$ we get the desired result 83 . 
Proof of Lemma 7.1. Observe that, with our usual notations, $\tilde{\pi}=\pi_{D_{u}}^{\gamma_{i, j-1}^{(L)}}$ and

$$
\hat{\pi}=\tilde{\pi}\left(\cdot \mid \cdot \geq \gamma_{i, j-1}^{(L)}-L^{-1+\delta / 40}\right),
$$

from which it is immediate to deduce

$$
\|\hat{\pi}-\tilde{\pi}\| \leq \frac{\tilde{\pi}\left(h \nsucceq \gamma_{i, j-1}^{(L)}-L^{-1+\delta / 40}\right)}{1-\tilde{\pi}\left(h \nsucceq \gamma_{i, j-1}^{(L)}-L^{-1+\delta / 40}\right)},
$$

where $h \nsucceq \gamma_{i, j-1}^{(L)}-L^{-1+\delta / 40}$ (violation of the floor constraint) is the event that there exists $v \in D_{u}$ such that $h(v)<\gamma_{i, j-1}^{(L)}-L^{-1+\delta / 40}$.

It is not hard to see that $\tilde{\pi}\left(h \nsucceq \gamma_{i, j-1}^{(L)}-L^{-1+\delta / 40}\right)$ is $O\left(L^{-n}\right)$ for any given $n$. Indeed, analogously to 88 one has that, for $v \in \partial D_{u}$,

$$
\gamma_{i, j-1}^{(L)}(v) \geq \pi_{L}^{A B C}(h(v))-C_{3} \epsilon_{L} L^{-1+\delta / 50} .
$$

From Proposition 3.5 one deduces that, except with $\tilde{\pi}$-probability $O\left(L^{-n}\right)$,

$$
h(v) \geq \pi_{L}^{A B C}(h(v))-(1 / 2) L^{-1+\delta / 40} \quad \text { for every } \quad v \in D_{u} .
$$

On the other hand, from 88 we see that for every $v \in D_{u}$

$$
\gamma_{i, j-1}^{(L)}(v)-L^{-1+\delta / 40} \leq \pi_{L}^{A B C}(h(v))-(3 / 4) L^{-1+\delta / 40} .
$$

\section{Proof of Corollary 3.2}

Let

$$
\epsilon^{(n)}=\max _{x \in \partial U}\left|\varphi(x)-\varphi^{(n)}(x)\right|
$$

and set

$$
\tilde{\varphi}^{(n)}=\varphi^{(n)}-\min _{x \in \partial U}\left(\varphi^{(n)}(x)-\varphi(x)\right)
$$

(we are just adding a constant to the boundary height $\varphi^{(n)}$ ). Note that

$$
\varphi \leq \tilde{\varphi}^{(n)} \leq \varphi+2 \epsilon^{(n)}
$$

so that, if $\tilde{\phi}^{(n)}$ is the macroscopic shape in $U$ with boundary height $\tilde{\varphi}^{(n)}$, one has

$$
\bar{\phi} \leq \tilde{\phi}^{(n)} \leq \bar{\phi}+2 \epsilon^{(n)} .
$$

If $h_{t}^{\eta}$ and $\tilde{h}_{t}^{\sigma}$ denote respectively the dynamics with boundary heights $\varphi, \tilde{\varphi}^{(n)}$ and initial conditions $\eta, \sigma$, one has by monotonicity

$$
\begin{aligned}
\max _{\eta} \sup _{t>c(\delta) L^{2+\delta}} \mathbb{P}\left(\exists v \in U_{L}: h_{t}^{\eta}(v)>\bar{\phi}(v)+3 \epsilon^{(n)}\right) \\
\leq \max _{\sigma} \sup _{t>c(\delta) L^{2+\delta}} \mathbb{P}\left(\exists v \in U_{L}: \tilde{h}_{t}^{\sigma}(v)>\bar{\phi}(v)+3 \epsilon^{(n)}\right) \\
\quad \leq \max _{\sigma} \sup _{t>c(\delta) L^{2+\delta}} \mathbb{P}\left(\exists v \in U_{L}: \tilde{h}_{t}^{\sigma}(v)>\tilde{\phi}^{(n)}(v)+\epsilon^{(n)}\right)
\end{aligned}
$$

where we used $(92)$ in the second step. Theorem 3.1 says that the third line of $(93)$ is some function $f(n, L)$ that vanishes as $L \rightarrow \infty$. It is then standard that one can choose some sequence $n(L)$ that diverges as $L \rightarrow \infty$ for which 
$f(n(L), L)$ still converges to zero. In conclusion, setting $\epsilon_{L}:=\epsilon^{(n(L))}$, that tends to zero as $L \rightarrow \infty$, we have proven

$$
\max _{\eta} \sup _{t>c(\delta) L^{2+\delta}} \mathbb{P}\left(\exists v \in U_{L}: h_{t}^{\eta}(v)>\bar{\phi}(v)+3 \epsilon_{L}\right)=o(1)
$$

which is "half" of the claim (11). The other bound can be obtained similarly.

Appendix A. Proof of Eq. 12

A.1. Mean height. We have to compute the average height difference between two lattice points $u$ and $v$ in $\mathcal{T}_{L} \cap \bigcirc_{a b c}$. Recall Remark 2.5. the height difference is directly related to the number of lozenges crossed by a lattice path from $u$ to $v$ (the height difference is independent of the chosen path). We will assume that the vector $u-v$ is along one of the three lattice directions of $\mathcal{T}_{L}$ (in the general case, we can always reduce to this situation by choosing the path from $u$ to $v$ as a concatenation of a $L$-independent number of straight paths along these directions) and by symmetry we consider only the case where $u-v$ is in the vertical direction, with $v$ above $u$.

To avoid a plethora of $\lfloor\cdot\rfloor$, let us assume that

$$
A=a L, B=b L, C=c L
$$

are even integers. Recall our choice of (non-orthogonal) coordinates $(x, y)$ for points in $\mathcal{T}_{L}$. In this Appendix, to fit better the notation of [21], it is convenient to translate the origin of the coordinates (that used to be in the center of the hexagon until now) in such a way that the center of the hexagon $\bigcirc_{a_{L} b_{L} c_{L}}$ has coordinates

$$
(-a-(b+c) / 2+1 /(2 L),-(3 / 2) a-c-b / 2+1 /(2 L)) .
$$

See Figure 4. Note that we translated the origin by a half-integer number of lattice steps in both directions $x$ and $y$. Given a vertical edge in $\mathcal{T}_{L}$, let us label it with the coordinates $(x, y)$ (that are now integers times $1 / L$ ) of its mid-point. Again to stay closer to the notations of [21], here we assume that $a+c=1$, instead of our usual normalization $a+b+c=1$.

Recall that $u$ and $v$ are points in $\mathcal{T}_{L}$ related by a vertical segment and let $p_{i}, i=0, \ldots, n-1$ be the vertical lattice edges composing such segment (labeled say from below). Clearly, $n=L|v-u|$ (because each edge of $\mathcal{T}_{L}$ has length $1 / L)$. In this case from Remark 2.5 we see that

$$
\pi_{L}^{a b c}(h(v))-\pi_{L}^{a b c}(h(u))=\frac{1}{L} \sum_{i=0}^{n-1}\left[1-\pi_{L}^{a b c}\left(\mathbf{1}_{p_{i}}\right)\right]
$$

with $\mathbf{1}_{p}$ the indicator function that the vertical edge $p$ crosses a horizontal lozenge. We will prove the following estimate (which is a special case of estimates proved in [21, Section 7], except for the explicit control of the error term):

Proposition A.1. Let $p=(x, y)$ be a vertical edge contained in $\bigcirc_{a b c}$. One has

$\pi_{L}^{a b c}\left(\mathbf{1}_{p}\right)=\Pi_{\infty}(x, y)+O(1 / L):=\frac{1}{2 \pi i} \int_{\overline{\mathrm{w}}_{c}}^{\mathrm{w}_{\mathrm{c}}} \frac{(1+y-x)}{(z+x)(z+y+1)} d z+O(1 / L)(96)$ where: 
- the $O(1 / L)$ error is uniform for $(x, y)$ in compact subsets of $\bigcirc_{a b c}$;

- $\mathrm{w}_{\mathrm{c}}=\mathrm{w}_{\mathrm{c}}(x, y, a, b, c)$ is the unique non-real critical point (w.r.t. $\left.w\right)$ of the function $S$, defined in equation (101), in the upper half complex plane and $\overline{\mathrm{w}}_{c}$ is the complex conjugate of $\mathrm{w}_{c}$;

- the contour of integration in the complex plane intersects the real axis to the right of both poles.

Now we can plug (96) into (95). After summation on $i$, the error term $O(1 / L)$ gives an error term $O\left(n / L^{2}\right)=O(|u-v| / L)$. The main term $1-\Pi_{\infty}$ gives as dominant term the line integral

$$
\int_{\mathcal{C}(u, v)}\left(1-\Pi_{\infty}\right) d s
$$

(with $\mathcal{C}(u, v)$ the straight path from $u$ to $v$ ) plus again an error $O(|u-v| / L)$ by Riemann approximation.

Finally we do not need to check that the line integral equals $\bar{\phi}_{a b c}(v)-$ $\bar{\phi}_{a b c}(u)$ : this follows from Theorem 3.3 on the existence of the limiting shape.

A.2. Correlation kernel. The proof of Proposition A.1 is essentially identical to the proof of Theorem 2 of [21, Section 7], except that we keep track more precisely of the size of errors. Actually, Theorem 2 of 21 considers a much more general situation: first of all, the domain to be tiled is not simply a hexagon but a more general polygonal shape (the hexagon being a particular case). Secondly, Theorem 2 of [21] allows to get the asymptotics of the probability of any event involving a fixed number $m$ of lozenges (say, the probability of the event $\left.\mathbf{1}_{p_{1}} \cdots \mathbf{1}_{p_{m}}\right)$. For simplicity of exposition, we will however re-

\begin{tabular}{|c|c|}
\hline \hline Our notations & Notations from [21] \\
\hline \hline$-1 / 2$ & $A_{1}$ \\
$A$ & $B_{1}-A_{1}$ \\
$B$ & $A_{2}-B_{1}$ \\
$C$ & $B_{2}-A_{2}$ \\
$x$ & $-x$ \\
$y$ & $-x-n$ \\
$L$ & $N$ \\
\hline
\end{tabular}

TABle 1 . The correspondence between the two sets of notations, in the case where in [21] the origin is chosen such that $A_{1}=$ $-1 / 2$. strict ourselves to the hexagonal region $\bigcirc_{a_{L} b_{L} c_{L}}$ (which corresponds to the polygonal shape of [21] with the choice $k=2$ there) and to the observable $\mathbf{1}_{p}$ we are interested in.

Let us introduce the Pochhammer symbol $(y)_{m}=y(y+1) \ldots(y+m-1)$. The probability that the vertical edge $p=(x, y)$ crosses a horizontal lozenge can be read from formulas (2.5) and (2.6) of [21]: it is given by a double integral in the complex plane, with a $L$-dependent but explicit kernel. In our language, we have:

Theorem A.2. Let $p=(x, y)$ be a vertical edge in the hexagon. Define $X=L x, Y=L y(X, Y$ are automatically integers with the present convention 
for coordinates). One has

$$
\begin{aligned}
& \pi_{L}^{a b c}\left(\mathbf{1}_{p}\right)=\frac{(L-X+Y)}{(2 i \pi)^{2}} \\
& \quad \times \oint d Z \oint d W \frac{(Z+X+1)_{L-X+Y-1}}{(W+X)_{L-X+Y+1}} \frac{1}{W-Z} \frac{(-W)_{A}}{(-Z)_{A}} \frac{(A+B-W)_{C}}{(A+B-Z)_{C}}
\end{aligned}
$$

where the integration contours on $Z$ and $W$ have to chosen such that:

- The $Z$ contour runs counter-clockwise and includes the integer real points $-X,-X+1, \ldots, A+B+C$ and no other integer point of the real line;

- The $W$ contour runs counter-clockwise; it contains the $Z$ contour and also integer points $-Y-L,-Y-L+1, \ldots,-X$ (it may contain other integer points).

We warn the reader who would like to find this formula in 21] that conventions in [21] are different from ours: the correspondence between the two sets of notations is conceptually trivial but a bit tricky as we need to rotate the hexagon and make an affine transformation to go from one setting to the other (compare for instance the shape of lozenges in our Figure 2 and in Figure 3 of [21]). We will not give details on the transformation but we summarize the correspondence in Table 1 .

A.3. Contour changes. In this section we use upper-case letters for lengths and coordinates proportional to $L$ and lower-case letters for the corresponding rescaled variables of order 1.

First we change the scale by the change of variable $Z \rightarrow Z / L=z$ and $W \rightarrow W / L=w$ and we reorder the resulting $L$-dependent factor to get:

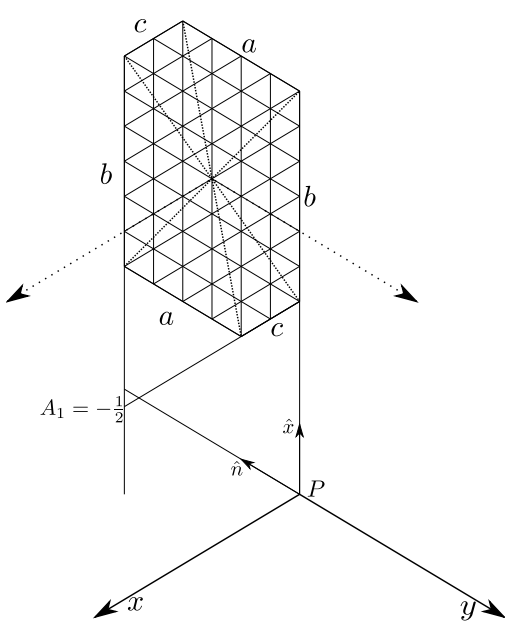

Figure 4. The coordinates we used until now (dotted axes) had the origin at the center of the hexagon. The coordinates we use in the Appendix are centered in $P$. The axes $\hat{n}, \hat{x}$ correspond to the the coordinates used in [21].

$$
\pi_{L}^{a b c}\left(\mathbf{1}_{p}\right)=\frac{1}{(2 \pi i)^{2}} \oint \oint \frac{1}{w-z} \frac{(1+y-x)}{(w+x)(w+y+1)} \frac{P_{L}(w, x, y)}{P_{L}(z, x, y)} d z d w
$$

where

$$
P_{L}(z, x, y)=\frac{(L+L y-L x-1) !}{(L z+L x+1)_{L+Y-X-1}}(-L z)_{A}(A+B-L z)_{C} .
$$




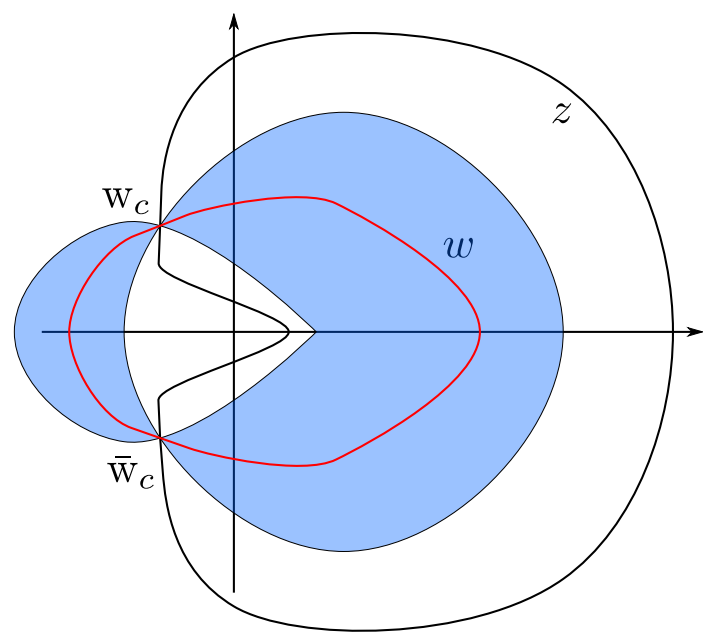

Figure 5. A schematic view of the new contours for $z$ and $w$. The shaded region corresponds to $\left\{w \in \mathbb{C}: \mathfrak{R}\left(S(w)-S\left(\mathrm{w}_{c}\right)\right)<\right.$ 0\}. See also [21, Fig. 11].

The following approximation result can be extracted from 21, Lemma 7.4]:

$$
P_{L}(w, x, y)=C_{L}\left(\frac{(w+x)(w+y+1)}{1+y-x}\right)^{1 / 2} \exp (L S(w ; x, y)+O(1 / L))
$$

where $C_{L}$ may depend on $A, B, C$ but not on $w, x, y$,

$$
\begin{aligned}
& S(w ; x, y)=(w+x) \ln (w+x)-(w+y+1) \ln (w+y+1) \\
&+(1+y-x) \ln (1+y-x)+(a-w) \ln (a-w)+(a+b+c-w) \ln (a+b+c-w) \\
&-(-w) \ln (-w)-(a+b-w) \ln (a+b-w) \quad(101)
\end{aligned}
$$

and the $O(1 / L)$ terms can be taken uniform on the integration contours.

As proven in [21], if $(x, y)$ is in the ellipse $\bigcirc_{a b c}$ then $S$ has two conjugate non-real critical points $\mathrm{w}_{c}, \overline{\mathrm{w}}_{c}$ (say with $\mathrm{w}_{c}$ in the upper half complex plane). Moreover, $S^{\prime \prime}\left(\mathrm{w}_{c} ; x, y\right) \neq 0$.

Recall that the integration contour for $w$ includes that for $z$. As explained in [21]:

Proposition A.3. One can move the integration contours so that:

- they cross exactly at the two points $\mathrm{w}_{c}, \overline{\mathrm{w}}_{c}$;

- the $w$ contour lies in the region of the complex plane where $\mathfrak{R}(S(w ; x, y)$ $\left.S\left(\mathrm{w}_{c} ; x, y\right)\right)<0$ (except at $\mathrm{w}_{c}, \overline{\mathrm{w}}_{c}$ where the real part is obviously zero);

- the $z$ contour lies in the region of the complex plane where $\mathfrak{R}(S(z ; x, y)$ $\left.S\left(\mathrm{w}_{c} ; x, y\right)\right)>0$ (except at $\mathrm{w}_{c}, \overline{\mathrm{w}}_{c}$ where it is zero);

- the contours avoid any poles of the integrand (that are on the real axis).

See Figure 5. Of course, in the process of moving the contours some residue will appear, because there is a pole $1 /(w-z)$ and the new contours 
cross. As shown in [21, Lemma 7.9], the residue is exactly the single integral

$$
\frac{1}{2 \pi i} \int_{\overline{\mathrm{w}}_{c}}^{\mathrm{w}_{c}} \frac{(1+y-x)}{(z+x)(z+y+1)} \frac{P_{L}(z, y, x)}{P_{L}(z, y, x)} d z=\frac{1}{2 \pi i} \int_{\overline{\mathrm{w}}_{c}}^{\mathrm{w}_{c}} \frac{(1+y-x)}{(z+x)(z+y+1)} d z
$$

which is nothing but $\Pi_{\infty}(x, y)$. To prove $(96)$ it remains therefore only to show that the double integral in the r.h.s. of (99), where now $z$ and $w$ run along the new contours, gives a contribution $O(1 / L)$.

A.4. Integral approximations. For the double integral we use 100 to get

$$
\begin{aligned}
\frac{1}{(2 i \pi)^{2}} \oint & \oint\left(\frac{(1+y-x)}{(z+x)(z+y+1)}\right)^{\frac{1}{2}}\left(\frac{(1+y-x)}{(w+x)(w+y+1)}\right)^{\frac{1}{2}} \\
& \times \frac{1}{w-z} \exp L[S(w ; x, y)-S(z ; x, y)](1+O(1 / L)) d z d w
\end{aligned}
$$

Recall the choice of the integration contours for $z$ and $w$ described in Proposition A.3 and observe that along such contours the exponential in (102) is bounded by 1 in absolute value. Also, we choose the contours so that close to the critical points they are exactly linear.

We fix some small $\delta>0$ and we divide the integral into three regions:

Region $1 w, z$ are both within distance $L^{-1 / 2+\delta}$ from $\mathrm{w}_{c}$ or both within distance $L^{-1 / 2+\delta}$ from $\overline{\mathrm{w}}_{c}$;

Region $2 z$ is within distance $L^{-1 / 2+\delta}$ from $\mathrm{w}_{c}$ and $w$ is within distance $L^{-1 / 2+\delta}$ from $\overline{\mathrm{w}}_{c}$, or viceversa;

Region 3 the rest of the integration contours.

Let us consider Region 3 first, and assume that $z$ is at distance at least $L^{-1 / 2+\delta}$ from both critical points. The first two factors in the integral are bounded (because the contours stay away from the poles). The factor $1 /(w-$ $z$ ) can be upper bounded by $L^{1 / 2-\delta}$ in absolute value. The exponential is $O\left(\exp \left(-L^{2 \delta}\right)\right)$. Indeed, recall that $\mathfrak{R}\left(S(w ; x, y)-S\left(\mathrm{w}_{c} ; x, y\right)\right) \leq 0$ while $\mathfrak{R}\left(S(z ; x, y)-S\left(\mathrm{w}_{c} ; x, y\right)\right)>0$. More precisely, since $S^{\prime \prime}\left(\mathrm{w}_{\mathrm{c}}, x, y\right) \neq 0$ and the third derivative is finite, one has $\mathfrak{R}\left(S(z ; x, y)-S\left(\mathrm{w}_{c} ; x, y\right)\right)>L^{-1+2 \delta}$ from a Taylor expansion. Finally the $O(1 / L)$ term in $(102)$ is bounded on the contour so it gives negligible contribution. In conclusion, the contribution from Region 3 is $O\left(\exp \left(-L^{2 \delta}\right)\right)$.

Now let us consider Region $\mathbf{2}$ and assume by symmetry that $w$ is close to $\mathrm{w}_{c}$ and $z$ is close to $\overline{\mathrm{w}}_{c}$. We can write $\mathfrak{R}\left(S(w ; x, y)-S\left(\mathrm{w}_{c} ; x, y\right)\right)=-C_{1} \mid w-$ $\left.\mathrm{w}_{c}\right|^{2}+O\left(\left|w-\mathrm{w}_{c}\right|^{3}\right), \mathfrak{R}\left(S(z ; x, y)-S\left(\mathrm{w}_{c} ; x, y\right)\right)=-C_{2}\left|z-\overline{\mathrm{w}}_{c}\right|^{2}+O\left(\left|z-\overline{\mathrm{w}}_{c}\right|^{3}\right)$ for some $C_{i}>0$. Furthermore since $z$ and $w$ are far from each other we can use a uniform bound on all terms outside the exponential and them we get a product of two Gaussian integrals, each of which gives a $O(1 / \sqrt{L})$ contribution. Again the $O(1 / L)$ term does not play any role.

Finally we consider Region 1 which is more difficult because the $\frac{1}{w-z}$ term is singular. By symmetry we will only consider the case with both $w$ and $z$ close to $\mathrm{w}_{c}$. Recall that the integration contours are linear near $\mathrm{w}_{c}$, i.e. one has

$$
w=\mathrm{w}_{c}+t \theta, t \in\left[-L^{-1 / 2+\delta}, L^{-1 / 2+\delta}\right], \quad z=\mathrm{w}_{c}+s \zeta, s \in\left[-L^{-1 / 2+\delta}, L^{-1 / 2+\delta}\right]
$$


with $\theta, \zeta$ two modulus- 1 complex numbers.

The expression we have to control is then:

$$
\begin{aligned}
E & =\int_{\mathrm{w}_{c}-\theta L^{-1 / 2+\delta}}^{\mathrm{w}_{c}+\theta L^{-1 / 2+\delta}} d w \int_{\mathrm{w}_{c}-\zeta L^{-1 / 2+\delta}}^{\mathrm{w}_{c}+\zeta L^{-1 / 2+\delta}} d z\left(\frac{(1+y-x)}{(z+x)(z+y+1)}\right)^{\frac{1}{2}} \\
& \times\left(\frac{(1+y-x)}{(w+x)(w+y+1)}\right)^{\frac{1}{2}} \frac{1+O(1 / L)}{w-z} \exp \{L[S(w ; x, y)-S(z ; x, y)]\} .
\end{aligned}
$$

The first step is to get rid of the $\left(\frac{(1+y-x)}{(z+x)(z+y+1)}\right)^{\frac{1}{2}}\left(\frac{(1+y-x)}{(w+x)(w+y+1)}\right)^{\frac{1}{2}}$ factor. Remark that it has a non-zero limit when $w=z=\mathrm{w}_{c}$ and that we can use a Taylor expansion to approximate it by $a_{0}+a_{1}\left(z-\mathrm{w}_{c}\right)+a_{2}\left(w-\mathrm{w}_{c}\right)+O\left(L^{-1+2 \delta}\right)$. Plugging this into the integral we get an expression of the form :

$$
E=\iint d w d z \frac{a_{0}+a_{1}\left(z-\mathrm{w}_{c}\right)+a_{2}\left(w-\mathrm{w}_{c}\right)+O\left(L^{-1+2 \delta}\right)}{w-z} \times \exp L[S(w ; x, y)-S(z ; x, y)]
$$

where from now on $\iint d w d z$ means $\int_{\mathrm{w}_{c}-\theta L^{-1 / 2+\delta}}^{\mathrm{w}_{c}+\theta L^{-1 / 2+\delta}} d w \int_{\mathrm{w}_{c}-\zeta L^{-1 / 2+\delta}}^{\mathrm{w}_{c}+\zeta L^{-1 / 2+\delta}} d z$. Note that the $O(1 / L)$ term has been absorbed into the $O\left(L^{-1+2 \delta}\right)$.

We start by looking at the most difficult term, the one proportional to $a_{0}$, call it $E_{0}$. Remark that we have :

$$
\iint d w d z \frac{1}{w-z} \exp \left\{L S^{\prime \prime}\left(\mathrm{w}_{c}\right)\left[\left(w-\mathrm{w}_{c}\right)^{2}-\left(z-\mathrm{w}_{c}\right)^{2}\right]\right\}=0 .
$$

Indeed the integrand is an odd function of $\left(z-\mathrm{w}_{c}, w-\mathrm{w}_{c}\right)$ and we integrate on a symmetric domain (the integral is absolutely convergent). We can thus rewrite $E_{0}$ as

$$
\begin{aligned}
& E_{0}=a_{0} \iint d w d z \frac{1}{w-z} \exp \left\{L S^{\prime \prime}\left(\mathrm{w}_{c}\right)\left[\left(w-\mathrm{w}_{c}\right)^{2}-\left(z-\mathrm{w}_{c}\right)^{2}\right]\right\} \\
\times & \left(-1+\exp \left\{L\left[S(w ; x, y)-S(z ; x, y)-S^{\prime \prime}\left(\mathrm{w}_{c}\right)\left(\left(w-\mathrm{w}_{c}\right)^{2}-\left(z-\mathrm{w}_{c}\right)^{2}\right)\right]\right\}\right) .
\end{aligned}
$$

With a Taylor expansion on $\exp L S(w)$ we get

$$
\begin{aligned}
E_{0}=a_{0} \iint d w d z \frac{O\left(L\left|w-\mathrm{w}_{c}\right|^{3}+L\left|z-\mathrm{w}_{c}\right|^{3}\right)}{w-z} \\
\times \exp \left\{L S^{\prime \prime}\left(\mathrm{w}_{c}\right)\left[\left(w-\mathrm{w}_{c}\right)^{2}-\left(z-\mathrm{w}_{c}\right)^{2}\right]\right\} .
\end{aligned}
$$

Remark that this expansion is valid because $L\left|w-\mathrm{w}_{c}\right|^{3}=O\left(L^{-1 / 2+3 \delta}\right)=$ $o(1)$. We take the absolute value inside the integral and change variables $\tilde{z}=\sqrt{L}\left(z-\mathrm{w}_{c}\right)$ and $\tilde{w}=\sqrt{L}\left(w-\mathrm{w}_{c}\right)$ to get

$$
\left|E_{0}\right| \leqslant \frac{K}{L} \int_{-\theta L^{\delta}}^{\theta L^{\delta}} d \tilde{w} \int_{-\zeta L^{\delta}}^{\zeta L^{\delta}} d \tilde{z} \frac{|\tilde{z}|^{3}+|\tilde{w}|^{3}}{|\tilde{z}-\tilde{w}|} \exp \Re\left[S^{\prime \prime}\left(\mathrm{w}_{c}\right)\left(\tilde{w}^{2}-\tilde{z}^{2}\right)\right]
$$

and we emphasize that both $S^{\prime \prime}\left(\mathrm{w}_{c}\right) \tilde{w}^{2}$ and $-S^{\prime \prime}\left(\mathrm{w}_{c}\right) \tilde{z}^{2}$ have negative real part. Finally since the linear integration contours for $\tilde{z}$ and $\tilde{w}$ are not colinear, we can lower bound $|\tilde{z}-\tilde{w}|$ by a constant times either $|\tilde{z}|$ or $|\tilde{w}|$ so we have

$$
\left|E_{0}\right| \leqslant \frac{K^{\prime}}{L} \int_{-\theta L^{\delta}}^{\theta L^{\delta}} d \tilde{w} \int_{-\zeta L^{\delta}}^{\zeta L^{\delta}} d \tilde{z}\left(|\tilde{z}|^{2}+|\tilde{w}|^{2}\right) \exp \Re\left[S^{\prime \prime}\left(\mathrm{w}_{c}\right)\left(\tilde{w}^{2}-\tilde{z}^{2}\right)\right]
$$


and the Gaussian integral in the right hand side is bounded uniformly in $L$.

Let us go back to the integral $E$. The terms $E_{1}, E_{2}$ containing $a_{1}$ or $a_{2}$ can be treated similarly to $E_{0}$. They are actually easier since $\left(w-\mathrm{w}_{c}\right)$ or $\left(z-\mathrm{w}_{c}\right)$ produce a term $L^{-1 / 2} \tilde{w}$ or $L^{-1 / 2} \tilde{z}$ : since $\tilde{w} /(\tilde{w}-\tilde{z})$ is bounded, one gets for $i=1,2$

$$
\left|E_{i}\right| \leqslant \frac{K^{\prime \prime}\left|a_{i}\right|}{L} \int_{-\theta L^{\delta}}^{\theta L^{\delta}} d \tilde{w} \int_{-\zeta L^{\delta}}^{\zeta L^{\delta}} d \tilde{z} \exp \left\{\Re\left[S^{\prime \prime}\left(\mathrm{w}_{c}\right)\left(\tilde{w}^{2}-\tilde{z}^{2}\right)\right]\right\}(1+o(1))=O(1 / L) .
$$

Finally, the term $E_{R}$ containing the $O\left(L^{-1+2 \delta}\right)$ error can be estimated once again with the same Taylor expansion of $S$ and the same change of variables, and it turns out to be $O\left(L^{-3 / 2+2 \delta}\right)$. We just have to remark that the integral

$$
\int_{-\theta L^{\delta}}^{\theta L^{\delta}} d \tilde{w} \int_{-\zeta L^{\delta}}^{\zeta L^{\delta}} d \tilde{z} \frac{1}{|\tilde{z}-\tilde{w}|} \exp \Re\left[S^{\prime \prime}\left(\mathrm{w}_{c}\right)\left(\tilde{w}^{2}-\tilde{z}^{2}\right)\right]
$$

does not diverge with $L$ since the singularity $\frac{1}{|\tilde{z}-\tilde{w}|}$ is integrable in $\mathbb{R}^{2}$ (recall that even though $\tilde{z}$ and $\tilde{w}$ are complex they live on different lines so we are really integrating on a two dimensional box) and thus the Gaussian integral is bounded.

Overall we have proven that the double integral 102$)$ is $O(1 / L)$.

\section{ACKNOWLEDGMENTS}

We are very grateful to Alexei Borodin and Leonid Petrov for precious indications on the methods of [21, 22], and to Pietro Caputo and Fabio Martinelli for many enlightening discussions. F. L. T. acknowledges the support of Agence Nationale de la Recherche through grant ANR-2010-BLAN-0108.

\section{REFERENCES}

[1] P. Caputo, F. Martinelli, F. L. Toninelli, Mixing times of monotone surfaces and SOS interfaces: a mean curvature approach, Comm. Math. Phys. 311 (2012), 157-189

[2] P. Caputo, F. Martinelli, F. Simenhaus, F. L. Toninelli, "Zero" temperature stochastic $3 D$ Ising model and dimer covering fluctuations: a first step towards interface mean curvature motion, Comm. Pure Appl. Math. 64 (2011), 778-831

[3] H. Cohn, M. Larsen, J. Propp, The Shape of a Typical Boxed Plane Partition, New York J. Math. 4 (1998), 137-165

[4] H. Cohn, R. Kenyon, J. Propp, A variational principle for domino tilings, J. Amer. Math. Soc. 14 (2001), 297-346

[5] N. Destainville, Flip dynamics in octagonal rhombus tiling sets, Phys. Rev. Lett. 88 (2002), 030601

[6] P. Diaconis, L. Saloff-Coste, Comparison theorems for reversible Markov chains, Ann. Appl. Probab. 3 (1993), 696-730

[7] M. Giaquinta, Multiple integrals in the calculus of variations and nonlinear elliptic systems, Ann. Math. Studies 105, Princeton Univ. Press (1983)

[8] W. B. Gordon, On the Diffeomorphisms of Euclidean Space, Amer. Math. Monthly 79 (1972), 755-759

[9] J. Hadamard. Sur les tranformations ponctuelles, Bull. Soc. Math. France, 34 (1906) 71-94; Oeuvres, pp. 349-363 and pp. 383-384

[10] C. L. Henley, Relaxation time for a dimer covering with height representation, J. Statist. Phys. 89 (1997), 483-507

[11] W. Jockusch, J. Propp, P. Shor, Random domino tilings and the arctic circle theorem, arXiv preprint math/9801068

[12] R. Kenyon, Conformal invariance of domino tiling, Ann. Probab. 28 (2000), 759-795 
[13] R. Kenyon, A. Okounkov, S. Sheffield, Dimers and amoebae, Ann. Math. 163 (2006), 1019-1056

[14] R. Kenyon, J. Propp, D. B. Wilson, Trees and matchings, Elec. J. Comb. 7 (2000), $\mathrm{R} 25$.

[15] H. Lacoin, F. Simenhaus, F. L. Toninelli, Zero-temperature 2D Ising model and anisotropic curve-shortening flow, to appear on J. Eur. Math Soc, arXiv:1112.3160

[16] H. Lacoin, F. Simenhaus, F. L. Toninelli, The heat equation shrinks Ising droplets to points, arXiv:1306.4507

[17] B. Laslier, F. L. Toninelli, How quickly can we sample a uniform domino tiling of the $2 L \times 2 L$ square?, arXiv:1210.5456

[18] B. Laslier, F. L. Toninelli, in preparation

[19] M. Luby, D. Randall, A. Sinclair, Markov Chain Algorithms for Planar Lattice Structures, SIAM J. Comput. 31 (2001), 167-192

[20] M. Mucha, P. Sankowski, Maximum matchings in planar graphs via Gaussian elimination, Algorithmica 45 (2006), 3-20.

[21] L. Petrov, Asymptotics of Random Lozenge Tilings via Gelfand-Tsetlin Schemes, arXiv:1202.3901

[22] L. Petrov, Asymptotics of Uniformly Random Lozenge Tilings of Polygons. Gaussian Free Field, to appear on Ann. Probab., arXiv:1206.5123

[23] H. Spohn, Interface motion in models with stochastic dynamics, J. Stat. Phys. 71 (1993), 1081-1132

[24] D. Randall, P. Tetali, Analyzing Glauber dynamics by comp arison of Markov chains, J. Math. Phys. 41 (2000), 1598-1615

[25] D. B. Wilson, Mixing times of Lozenge tiling and card shuffing Markov chains, Ann. Appl. Probab. 14 (2004), 274-325

[26] D. B. Wilson, Generating random spanning trees more quickly than the cover time, Proc. of the 28th annual ACM symposium on Theory of computing (STOC '96), pp. 296-303.

Institut Camille Jordan, Université Lyon 1, 43 BD DU 11 nOvembre 1918, 69622 Villeurbanne, France, E-Mail: LASLier@Math.uniV-Lyon1.Fr

CNRS and Institut Camille Jordan, Université Lyon 1, 43 BD DU 11 NOvembre 1918, 69622 Villeurbanne, France, E-MAil: TONinElli@MATH.UNiV-Lyon1.FR 